\title{
Ranks of twists of elliptic curves and Hilbert's tenth problem
}

\author{
B. Mazur • K. Rubin
}

Received: 26 April 2009 / Accepted: 26 April 2010 / Published online: 19 May 2010 (C) The Author(s) 2010. This article is published with open access at Springerlink.com

\begin{abstract}
In this paper we investigate the 2-Selmer rank in families of quadratic twists of elliptic curves over arbitrary number fields. We give sufficient conditions on an elliptic curve so that it has twists of arbitrary 2-Selmer rank, and we give lower bounds for the number of twists (with bounded conductor) that have a given 2-Selmer rank. As a consequence, under appropriate hypotheses we can find many twists with trivial Mordell-Weil group, and (assuming the Shafarevich-Tate conjecture) many others with infinite cyclic Mordell-Weil group. Using work of Poonen and Shlapentokh, it follows from our results that if the Shafarevich-Tate conjecture holds, then Hilbert's Tenth Problem has a negative answer over the ring of integers of every number field.
\end{abstract}

\section{Introduction and main results}

In this paper we investigate the 2-Selmer rank in families of quadratic twists of elliptic curves over arbitrary number fields. We give sufficient conditions on an elliptic curve so that it has twists of 2-Selmer rank $r$ for every $r \geq 0$, and discuss other conditions under which the 2-Selmer ranks of all quadratic

This material is based upon work supported by the National Science Foundation under grants DMS-0700580 and DMS-0757807.

B. Mazur ( $\square)$

Department of Mathematics, Harvard University, Cambridge, MA 02138, USA

e-mail: mazur@math.harvard.edu

K. Rubin

Department of Mathematics, UC Irvine, Irvine, CA 92697, USA

e-mail: krubin@math.uci.edu 
twists have the same parity. We also give lower bounds for the number of twists (with bounded conductor) that have a given 2-Selmer rank.

Since the 2-Selmer rank is an upper bound for the Mordell-Weil rank, our results have consequences for the Mordell-Weil rank. Under appropriate hypotheses we can find many twists with trivial Mordell-Weil group, and (assuming the Shafarevich-Tate conjecture below) many others with infinite cyclic Mordell-Weil group.

Here are two applications of our results. The first settles an open question mentioned to us by Poonen.

Theorem 1.1 If $K$ is a number field, then there is an elliptic curve $E$ over $K$ with $E(K)=0$.

The second application combines our results with work of Poonen and Shlapentokh. It relies on a weak version of the Shafarevich-Tate conjecture, Conjecture $\mathrm{WT}_{2}(K)$ below.

Theorem 1.2 Suppose Conjecture $\mathrm{WT}_{2}(K)$ holds for every number field $K$. Then for every number field $K$, Hilbert's Tenth Problem is undecidable (i.e., has a negative answer) over the ring of integers of $K$.

We now discuss our methods and results in more detail. If $K$ is a number field and $E$ is an elliptic curve over $K$, let $\operatorname{Sel}_{2}(E / K)$ be the 2-Selmer group of $E / K$ (see Sect. 2 for the definition) and

$$
d_{2}(E / K):=\operatorname{dim}_{\mathbf{F}_{2}} \operatorname{Sel}_{2}(E / K) .
$$

Then $\operatorname{rank}(E(K)) \leq d_{2}(E / K)$, so

$$
d_{2}(E / K)=0 \Longrightarrow \operatorname{rank}(E(K))=0 .
$$

If $F / K$ is a quadratic extension, let $E^{F}$ denote the quadratic twist of $E$ by $F / K$. We will allow the "trivial quadratic extension" $F=K$, in which case $E^{F}=E$. For $X \in \mathbf{R}^{+}$define

$$
N_{r}(E, X):=\mid\left\{\text { quadratic } F / K: d_{2}\left(E^{F} / K\right)=r \text { and } \mathbf{N}_{K / \mathbf{Q}} \mathfrak{f}(F / K)<X\right\} \mid
$$

where $\mathfrak{f}(F / K)$ denotes the finite part of the conductor of $F / K$.

\subsection{Controlling the Selmer rank}

Not all elliptic curves have twists of every 2-Selmer rank. For example, some elliptic curves have "constant 2-Selmer parity", meaning that $d_{2}\left(E^{F} / K\right) \equiv$ $d_{2}(E / K)(\bmod 2)$ for all quadratic extensions $F / K$. A theorem of Dokchitser and Dokchitser [7, Theorem 1] (see Theorem 9.3 below), combined 
with standard conjectures, predicts that $E / K$ has constant 2-Selmer parity if and only if $K$ is totally imaginary and $E$ acquires everywhere good reduction over an abelian extension of $K$. See Sect. 9 for a discussion of the phenomenon of constant 2-Selmer parity, and some examples.

We expect that constant parity and the existence of rational 2-torsion are the only obstructions to having twists of every 2-Selmer rank. We also expect that $N_{r}(E, X)$ should grow like a positive constant times $X$, whenever it is nonzero. Namely, we expect the following.

Conjecture 1.3 Suppose $K$ is a number field and $E$ is an elliptic curve over $K$.

(i) If $r \geq \operatorname{dim}_{2} E(K)[2]$ and $r \equiv d_{2}(E / K)(\bmod 2)$, then $N_{r}(E, X) \gg X$.

(ii) If $K$ has a real embedding, or if $E / K$ does not acquire everywhere good reduction over an abelian extension of $K$, then $N_{r}(E, X) \gg X$ for every $r \geq \operatorname{dim}_{\mathbf{F}_{2}} E(K)[2]$.

When $K=\mathbf{Q}$ and $E$ is $y^{2}=x^{3}-x$, Heath-Brown [10] has shown that $\lim _{X \rightarrow \infty} N_{r}(E, X) / X=\alpha_{r}$ for every $r \geq 2$, with an explicit positive constant $\alpha_{r}$. Related results have been obtained by Swinnerton-Dyer [27] when $K=\mathbf{Q}$ and $E$ is an elliptic curve with all 2-torsion points rational.

In the direction of Conjecture 1.3, we have the following results.

Theorem 1.4 Suppose $K$ is a number field, $E$ is an elliptic curve over $K$, $r \geq 0$, and $E$ has a quadratic twist $E^{\prime} / K$ with $d_{2}\left(E^{\prime} / K\right)=r$. Then:

(i) If $\mathrm{Gal}(K(E[2]) / K) \cong S_{3}$, then $N_{r}(E, X) \gg X /(\log X)^{2 / 3}$.

(ii) If $\operatorname{Gal}(K(E[2]) / K) \cong \mathbf{Z} / 3 \mathbf{Z}$, then $N_{r}(E, X) \gg X /(\log X)^{1 / 3}$.

Note that $\operatorname{Gal}(K(E[2]) / K)$ is isomorphic to $S_{3}$ or $\mathbf{Z} / 3 \mathbf{Z}$ if and only if $E(K)[2]=0$.

When $K=\mathbf{Q}$, a version of Theorem 1.4 was proved by Chang in [3, Theorem 4.10]. Also in the case $K=\mathbf{Q}$, Chang has proved (slightly weaker) versions of Theorem 1.7 and Corollary 1.12 below, namely [4, Theorem 1.1] and [4, Corollary 1.2], respectively.

In the statements below, we will use the phrase " $E$ has many twists" with some property to indicate that the number of such twists, ordered by $\mathbf{N}_{K / \mathbf{Q}} \mathfrak{f}(F / K)$, is $\gg X /(\log X)^{\alpha}$ for some $\alpha \in \mathbf{R}$.

Theorem 1.5 Suppose $K$ is a number field, and $E$ is an elliptic curve over $K$ such that $E(K)[2]=0$. Suppose further that either $K$ has a real embedding, or that $E$ has multiplicative reduction at some prime of $K$.

If $r=0,1$, or $r \leq d_{2}(E / K)$, then $E$ has many quadratic twists $E^{\prime} / K$ with $d_{2}\left(E^{\prime} / K\right)=r$. 
Theorem 1.6 Suppose $K$ is a number field, and $E$ is an elliptic curve over $K$ such that $\operatorname{Gal}(K(E[2]) / K) \cong S_{3}$. Let $\Delta_{E}$ be the discriminant of some model of $E$, and suppose further that $K$ has a place $v_{0}$ satisfying one of the following conditions:

- $v_{0}$ is real and $\left(\Delta_{E}\right)_{v_{0}}<0$, or

- $v_{0} \nmid 2 \infty, E$ has multiplicative reduction at $v_{0}$, and $\operatorname{ord}_{v_{0}}\left(\Delta_{E}\right)$ is odd.

Then for every $r \geq 0, E$ has many quadratic twists $E^{\prime} / K$ with $d_{2}\left(E^{\prime} / K\right)=r$.

Theorem 1.7 Suppose $K$ is a number field, and $E$ is an elliptic curve over $K$ such that $E(K)[2]=0$. If $0 \leq r \leq d_{2}(E / K)$ and $r \equiv d_{2}(E / K)(\bmod 2)$, then $E$ has many quadratic twists $E^{\prime} / K$ such that $d_{2}\left(E^{\prime} / K\right)=r$.

Corollary 1.8 Suppose $K$ is a number field, and $E$ is an elliptic curve over $K$ with constant 2-Selmer parity such that $\mathrm{Gal}(K(E[2]) / K) \cong S_{3}$. Let $j(E)$ be the $j$-invariant of $E$, and suppose further that $j(E) \neq 0$ and $K$ has an archimedean place $v$ such that $(j(E))_{v} \in \mathbf{R}$ and $(j(E))_{v}<1728$. Then for every $r \equiv d_{2}(E / K)(\bmod 2), E$ has many quadratic twists $E^{\prime} / K$ such that $d_{2}\left(E^{\prime} / K\right)=r$.

For every number field $K$, there are elliptic curves $E$ over $K$ satisfying the hypotheses of Theorem 1.6. In fact, $E$ can be taken to be the base change of an elliptic curve over $\mathbf{Q}$ (see Lemma 5.4).

Corollary 1.9 Suppose $K$ is a number field. There are elliptic curves $E$ over $K$ such that for every $r \geq 0, E$ has many twists $E^{\prime} / K$ with $d_{2}\left(E^{\prime} / K\right)=r$.

\subsection{Controlling the Mordell-Weil rank}

Using the relation between $d_{2}(E / K)$ and $\operatorname{rank}(E(K))$ leads to the following corollaries.

Corollary 1.10 Suppose $K$ is a number field, and $E$ is an elliptic curve over $K$ such that $E(K)[2]=0$. Suppose further that either $K$ has a real embedding, or that $E$ has multiplicative reduction at some prime of $K$. Then $E$ has many twists $E^{\prime} / K$ with $E^{\prime}(K)=0$.

When $K=\mathbf{Q}$, Corollary 1.10 was proved by Ono and Skinner ([19, Sect. 1], [18, Corollary 3]), using methods very different from ours (modularity and special values of $L$-functions).

Theorem 1.1 is an immediate consequence of the following corollary.

Corollary 1.11 Suppose $K$ is a number field. There are elliptic curves $E$ over $K$ such that $E$ has many twists $E^{\prime} / K$ with $E^{\prime}(K)=0$. 
If $E$ is an elliptic curve over a number field $K$, let $\amalg(E / K)$ denote the Shafarevich-Tate group of $E$ over $K$ (see Sect. 2). A conjecture that is part of the folklore (usually called the Shafarevich-Tate Conjecture $[1$, p. 239 , footnote (5)]) predicts that $\amalg(E / K)$ is finite. If the 2-primary subgroup $\amalg(E / K)\left[2^{\infty}\right]$ is finite, then the Cassels pairing shows that $\operatorname{dim}_{\mathbf{F}_{2}} \amalg(E / K)[2]$ is even. We record this 2-parity conjecture as follows.

Conjecture $\amalg_{2}(K)$ For every elliptic curve $E / K, \operatorname{dim}_{\mathbf{F}_{2}} \amalg(E / K)[2]$ is even.

Corollary 1.12 Suppose $K$ is a number field, and $E$ is an elliptic curve over $K$ such that $E(K)[2]=0$. Suppose further that either $K$ has a real embedding, or that $E$ has multiplicative reduction at some prime of $K$. If Conjecture $\mathrm{WT}_{2}(K)$ holds, then $E$ has many quadratic twists with infinite cyclic Mordell-Weil group.

Skorobogatov and Swinnerton-Dyer [26] obtained results related to Corollary 1.12 in the case where all the 2-torsion on $E$ is rational over $K$.

\subsection{Controlling the rank over two fields simultaneously}

Suppose $L / K$ is a cyclic extension of prime degree of number fields. With care, we can simultaneously control the 2-Selmer rank of twists of $E$ over $K$ and over $L$, leading to the following result.

Theorem 1.13 Suppose $L / K$ is a cyclic extension of prime degree of number fields. Then there is an elliptic curve $E$ over $K$ with $\operatorname{rank}(E(L))=$ $\operatorname{rank}(E(K))$.

If Conjecture $\mathrm{WT}_{2}(K)$ is true, then there is an elliptic curve $E$ over $K$ with $\operatorname{rank}(E(L))=\operatorname{rank}(E(K))=1$.

Assuming standard conjectures, the second assertion of Theorem 1.13 can fail when $L / K$ is not cyclic. See Remark 7.7 for more about this.

By using the final assertion of Lemma 5.4 in the proof of Theorem 1.13, we can take the elliptic curve $E$ in Theorem 1.13 to be a twist over $K$ of an elliptic curve defined over Q. Similarly, in Corollaries 1.9 and 1.11 we can conclude that there are elliptic curves $E / \mathbf{Q}$ that have many quadratic twists $E^{\prime} / K$ having $d_{2}\left(E^{\prime} / K\right)=r$ or $E^{\prime}(K)=0$, respectively.

Poonen and Shlapentokh showed how to use Theorem 1.13 together with ideas from [20, Theorem 1 and Corollary 2], [5], and [24] to prove Theorem 1.2 about Hilbert's Tenth Problem. In fact one can be more precise about how much of Conjecture $\mathrm{WT}_{2}$ is required; see Theorem 8.1.

A theorem of Eisenträger [8, Theorem 7.1] gives the following corollary of Theorem 1.2. 
Corollary 1.14 Suppose Conjecture $\mathrm{WT}_{2}(K)$ holds for every number field $K$. Then Hilbert's Tenth Problem has a negative answer over every infinite ring $A$ that is finitely generated over $\mathbf{Z}$.

\subsection{Some remarks about the proofs}

Our methods are different from the classical 2-descent, and are more in the spirit of the work of Kolyvagin, especially as described in [14]. If $F$ is a quadratic extension of $K$, the 2-Selmer group $\operatorname{Sel}_{2}\left(E^{F} / K\right)$ is defined as a subgroup of $H^{1}\left(K, E^{F}[2]\right)$ cut out by local conditions (see Definition 2.3). The $G_{K}$-modules $E[2]$ and $E^{F}$ [2] are canonically isomorphic, so we can view $\operatorname{Sel}_{2}\left(E^{F} / K\right) \subset H^{1}(K, E[2])$ for every $F$. In other words, all the different 2-Selmer groups are subgroups of $H^{1}(K, E[2])$, cut out by different local conditions. Our method is to try to construct $F$ so that the local conditions defining $\operatorname{Sel}_{2}(E / K)$ and $\operatorname{Sel}_{2}\left(E^{F} / K\right)$ agree everywhere except at most one place, and to use that one place to vary the 2-Selmer rank in a controlled manner.

For example, to prove Theorem 1.4 we find many different quadratic extensions $F$ for which all of the local conditions defining $\operatorname{Sel}_{2}(E / K)$ and $\operatorname{Sel}_{2}\left(E^{F} / K\right)$ are the same, so in fact $\operatorname{Sel}_{2}\left(E^{F} / K\right)=\operatorname{Sel}_{2}(E / K)$.

For another example, suppose the hypotheses of Theorem 1.6 are satisfied. We will take $F=\mathbf{Q}(\sqrt{\pi})$, where $\pi$ is a generator of a prime ideal $\mathfrak{p}$ chosen using the Cebotarev theorem, so that the local conditions defining $\operatorname{Sel}_{2}(E / K)$ and $\operatorname{Sel}_{2}\left(E^{F} / K\right)$ are the same for all places different from $\mathfrak{p}$. By choosing the prime $\mathfrak{p}$ appropriately, we will also ensure that $\operatorname{Sel}_{2}\left(E^{F} / K\right) \subset \operatorname{Sel}_{2}(E / K)$ with codimension one, so $d_{2}\left(E^{F} / K\right)=d_{2}(E / K)-1$.

Similarly, we can choose a different $F$ such that $\operatorname{Sel}_{2}(E / K) \subset \operatorname{Sel}_{2}\left(E^{F} / K\right)$ with codimension one, so $d_{2}\left(E^{F} / K\right)=d_{2}(E / K)+1$. Now Theorem 1.6 follows by induction.

Theorems 1.5, 1.7, and 1.13 are proved in the same general manner.

A key tool in several of our arguments is a theorem of Kramer [11, Theorem 1] that gives a formula for the parity of $d_{2}(E / K)+d_{2}\left(E^{F} / K\right)$ in terms of local data. See Theorem 2.7 below.

\subsection{Layout of the paper}

In the next section we define the 2-Selmer group and study the local subgroups that occur in the definition. In Sect. 3 we give a general result (Proposition 3.3) comparing the 2-Selmer ranks of quadratic twists, and lay the groundwork (Lemma 3.5) for using the Cebotarev theorem to construct useful twists.

Theorem 1.4 is proved in Sect. 4. Theorems 1.5, 1.6 and 1.7, and Corollaries 1.8, 1.9, 1.10, 1.11, and 1.12, are all proved in Sect. 5. In Sect. 6 we 
prove Theorem 1.13 in the case $[L: K]=2$, and the rest of Theorem 1.13 is proved in Sect. 7. Theorem 1.2 is proved in Sect. 8. In Sect. 9 we discuss elliptic curves with constant 2-Selmer parity.

\section{Local conditions}

Fix for this section a number field $K$.

Definition 2.1 Suppose $E$ is an elliptic curve over $K$. For every place $v$ of $K$, let $H_{\mathrm{f}}^{1}\left(K_{v}, E[2]\right)$ denote the image of the Kummer map

$$
E\left(K_{v}\right) / 2 E\left(K_{v}\right) \longrightarrow H^{1}\left(K_{v}, E[2]\right) .
$$

(Note that $H_{\mathrm{f}}^{1}\left(K_{v}, E[2]\right)$ depends on $E$, not just on the Galois module $E[2]$.)

\section{Lemma 2.2}

(i) If $v \nmid 2 \infty$ then $\operatorname{dim}_{\mathbf{F}_{2}}\left(H_{\mathrm{f}}^{1}\left(K_{v}, E[2]\right)\right)=\operatorname{dim}_{\mathbf{F}_{2}}\left(E\left(K_{v}\right)[2]\right)$.

(ii) If $v \nmid 2 \infty$ and $E$ has good reduction at $v$, then

$$
H_{\mathrm{f}}^{1}\left(K_{v}, E[2]\right) \cong E[2] /\left(\text { Frob }_{v}-1\right) E[2]
$$

with the isomorphism given by evaluating cocycles at the Frobenius automorphism $\operatorname{Frob}_{v}$.

Proof Suppose $v \nmid 2 \infty$, and let $\ell>2$ be the residue characteristic of $v$. Then $E\left(K_{v}\right)$ is a commutative profinite group with a pro- $\ell$ subgroup of finite index, so $H_{\mathrm{f}}^{1}\left(K_{v}, E[2]\right) \cong E\left(K_{v}\right) / 2 E\left(K_{v}\right)$ and $E\left(K_{v}\right)$ [2] are (finite dimensional) $\mathbf{F}_{2}$-vector spaces of the same dimension.

If in addition $E$ has good reduction at $v$, then (see for example [2])

$$
H_{\mathrm{f}}^{1}\left(K_{v}, E[2]\right)=H^{1}\left(K_{v}^{\mathrm{ur}} / K, E[2]\right) \subset H^{1}\left(K_{v}, E[2]\right)
$$

and (ii) follows.

Definition 2.3 Suppose $E$ is an elliptic curve over $K$. The 2-Selmer group $\mathrm{Sel}_{2}(E / K) \subset H^{1}(K, E[2])$ is the (finite) $\mathbf{F}_{2}$-vector space defined by the exactness of the sequence

$$
0 \longrightarrow \operatorname{Sel}_{2}(E / K) \longrightarrow H^{1}(K, E[2]) \longrightarrow \bigoplus_{v} H^{1}\left(K_{v}, E[2]\right) / H_{\mathrm{f}}^{1}\left(K_{v}, E[2]\right)
$$

The Kummer map $E(K) / 2 E(K) \rightarrow H^{1}(K, E[2])$ induces an exact sequence

$$
0 \longrightarrow E(K) / 2 E(K) \longrightarrow \operatorname{Sel}_{2}(E / K) \longrightarrow \amalg(E / K)[2] \longrightarrow 0
$$


where $\amalg(E / K)[2]$ is the kernel of multiplication by 2 in the ShafarevichTate group of $E / K$.

Recall that $d_{2}(E / K):=\operatorname{dim}_{\mathbf{F}_{2}} \operatorname{Sel}_{2}(E / K)$.

Remark 2.4 If $E$ is an elliptic curve over $K$ and $E^{F}$ is a quadratic twist, then there is a natural identification of Galois modules $E[2]=E^{F}$ [2]. This allows us to view $\operatorname{Sel}_{2}(E / K), \operatorname{Sel}_{2}\left(E^{F} / K\right) \subset H^{1}(K, E[2])$, defined by different sets of local conditions. By choosing $F$ carefully, we can ensure that the local conditions $H_{\mathrm{f}}^{1}\left(K_{v}, E[2]\right), H_{\mathrm{f}}^{1}\left(K_{v}, E^{F}[2]\right) \subset H^{1}\left(K_{v}, E[2]\right)$ coincide for all but at most one $v$, and then using global duality we will compare $d_{2}(E / K)$ and $d_{2}\left(E^{F} / K\right)$.

Lemma 2.5 If $F$ is a quadratic extension of $K$, then

$$
d_{2}(E / K)+d_{2}\left(E^{F} / K\right) \equiv d_{2}(E / F)+\operatorname{dim}_{\mathbf{F}_{2}}(E(F)[2])(\bmod 2) .
$$

Proof Let $\operatorname{Sel}_{2} \infty(E / K)$ denote the 2-power Selmer group of $E / K$, the direct limit over $n$ of the $2^{n}$-Selmer groups $\operatorname{Sel}_{2^{n}}(E / K)$ defined analogously to $\operatorname{Sel}_{2}(E / K)$ above. Using the Cassels pairing it is straightforward to show (see for example [15, Proposition 2.1])

$$
\operatorname{corank}_{\mathbf{Z}_{2}}\left(\operatorname{Sel}_{2} \infty(E / K)\right) \equiv d_{2}(E / K)+\operatorname{dim}_{\mathbf{F}_{2}} E(K)[2](\bmod 2) .
$$

The natural map

$$
\operatorname{Sel}_{2} \infty(E / K) \oplus \operatorname{Sel}_{2} \infty\left(E^{F} / K\right) \longrightarrow \operatorname{Sel}_{2} \infty(E / F)
$$

has finite kernel and cokernel, so

$$
\begin{aligned}
& \operatorname{corank}_{\mathbf{Z}_{2}}\left(\operatorname{Sel}_{2} \infty(E / K)\right)+\operatorname{corank}_{\mathbf{Z}_{2}}\left(\operatorname{Sel}_{2} \infty\left(E^{F} / K\right)\right) \\
& \quad=\operatorname{corank}_{\mathbf{Z}_{2}}\left(\operatorname{Sel}_{2} \infty(E / F)\right) .
\end{aligned}
$$

Combining this with (2), and observing that $E(K)[2] \cong E^{F}(K)[2]$, proves the congruence of the lemma.

Fix for the rest of this section an elliptic curve $E / K$ and a quadratic extension $F / K$. Recall that $E^{F}$ is the twist of $E$ by $F / K$. Let $\Delta_{E}$ be the discriminant of some model of $E$.

Definition 2.6 If $v$ is a place of $K$, let $E_{\mathbf{N}}\left(K_{v}\right) \subset E\left(K_{v}\right)$ denote the image of the norm map $E\left(F_{w}\right) \rightarrow E\left(K_{v}\right)$ for any choice of $w$ above $v$ (this is independent of the choice of $w$ ), and define

$$
\delta_{v}(E, F / K):=\operatorname{dim}_{\mathbf{F}_{2}}\left(E\left(K_{v}\right) / E_{\mathbf{N}}\left(K_{v}\right)\right) .
$$


The following theorem of Kramer will play an important role in many of our proofs below.

Theorem 2.7 (Kramer) We have

$$
d_{2}\left(E^{F} / K\right) \equiv d_{2}(E / K)+\sum_{v} \delta_{v}(E, F / K)(\bmod 2) .
$$

Proof This is a consequence of [11, Theorem 1]. Combining Theorems 1 and 2 of [11] shows that

$$
\operatorname{rank}(E(F))+\operatorname{dim}_{\mathbf{F}_{2}}(\amalg(E / F)[2]) \equiv \sum_{v} \delta_{v}(E, F / K)(\bmod 2) .
$$

By (1), the left-hand side of this congruence is $d_{2}(E / F)-\operatorname{dim}_{\mathbf{F}_{2}}(E(F)[2])$, and by Lemma 2.5 this is congruent to $d_{2}(E / K)+d_{2}\left(E^{F} / K\right)$.

Remark 2.8 A key step in Kramer's proof is the following remarkable construction. There are alternating Cassels pairings $h_{E}$ on $\operatorname{Sel}_{2}(E / K)$ and $h_{E^{F}}$ on $\operatorname{Sel}_{2}\left(E^{F} / K\right)$. Their sum is a new alternating pairing on the intersection $\operatorname{Sel}_{2}(E / K) \cap \operatorname{Sel}_{2}\left(E^{F} / K\right)$, and Kramer shows [11, Theorem 2] that the kernel of $h_{E}+h_{E^{F}}$ is $\mathbf{N}_{F / K} \operatorname{Sel}_{2}(E / F)$. Therefore

$$
\operatorname{dim}_{\mathbf{F}_{2}}\left(\operatorname{Sel}_{2}(E / K) \cap \operatorname{Sel}_{2}\left(E^{F} / K\right)\right) \equiv \operatorname{dim}_{\mathbf{F}_{2}}\left(\mathbf{N}_{F / K} \operatorname{Sel}_{2}(E / F)\right)(\bmod 2) .
$$

Lemma 2.9 Under the identification $H_{\mathrm{f}}^{1}\left(K_{v}, E[2]\right)=E\left(K_{v}\right) / 2 E\left(K_{v}\right)$, we have

$$
H_{\mathrm{f}}^{1}\left(K_{v}, E[2]\right) \cap H_{\mathrm{f}}^{1}\left(K_{v}, E^{F}[2]\right)=E_{\mathbf{N}}\left(K_{v}\right) / 2 E\left(K_{v}\right) .
$$

Proof This is [11, Proposition 7] or [15, Proposition 5.2] (the proof given in [15] works even if $p=2$, and even if $v \mid \infty$ ).

Lemma 2.10 (Criteria for equality of local conditions after twist) If at least one of the following conditions (i)-(v) holds:

(i) $v$ splits in $F / K$, or

(ii) $v \nmid 2 \infty$ and $E\left(K_{v}\right)[2]=0$, or

(iii) $E$ has multiplicative reduction at $v, F / K$ is unramified at $v$, and $\operatorname{ord}_{v}\left(\Delta_{E}\right)$ is odd, or

(iv) $v$ is real and $\left(\Delta_{E}\right)_{v}<0$, or

(v) $v$ is a prime where $E$ has good reduction and $v$ is unramified in $F / K$, then $H_{\mathrm{f}}^{1}\left(K_{v}, E[2]\right)=H_{\mathrm{f}}^{1}\left(K_{v}, E^{F}[2]\right)$ and $\delta_{v}(E, F / K)=0$. 
Proof By Lemma 2.9, we have

$$
\begin{aligned}
H_{\mathrm{f}}^{1}\left(K_{v}, E[2]\right)=H_{\mathrm{f}}^{1}\left(K_{v}, E^{F}[2]\right) & \Longleftrightarrow E_{\mathbf{N}}\left(K_{v}\right)=E\left(K_{v}\right) \\
& \Longleftrightarrow \delta_{v}(E, F / K)=0 .
\end{aligned}
$$

If $v$ splits in $F / K$ then $E_{\mathbf{N}}\left(K_{v}\right)=E\left(K_{v}\right)$.

If $v \nmid 2 \infty$ and $E\left(K_{v}\right)[2]=0$, then $H_{\mathrm{f}}^{1}\left(K_{v}, E[2]\right)=H_{\mathrm{f}}^{1}\left(K_{v}, E^{F}[2]\right)=0$ by Lemma 2.2(i).

If $E$ has multiplicative reduction at $v, F / K$ is unramified at $v$, and $\operatorname{ord}_{v}\left(\Delta_{E}\right)$ is odd, then [11, Propositions 1 and 2(a)] shows that $\delta_{v}(E, F / K)=0$.

If $v$ is real and $\left(\Delta_{E}\right)_{v}<0$, then $E\left(K_{v}\right)$ is connected and $\delta_{v}(E, F / K)=0$.

If $E$ has good reduction at $v$ and $v$ is unramified in $F / K$, then $\delta_{v}(E, F / K)=0$ by [13, Corollary 4.4]. This completes the proof.

Lemma 2.11 (Criterion for transversality of local conditions after twist) If $v \nmid 2 \infty, E$ has good reduction at $v$, and $v$ is ramified in $F / K$, then

$$
H_{\mathrm{f}}^{1}\left(K_{v}, E[2]\right) \cap H_{\mathrm{f}}^{1}\left(K_{v}, E^{F}[2]\right)=0, \quad \delta(E, F / K)=\operatorname{dim}_{\mathbf{F}_{2}}\left(E\left(K_{v}\right)[2]\right) .
$$

Proof For such v, [13, Corollary 4.6] or [15, Lemma 5.5] show that $E_{\mathbf{N}}\left(K_{v}\right)=2 E\left(K_{v}\right)$. Now the first assertion of the lemma follows from Lemma 2.9, and the second from Lemma 2.2(i).

\section{Comparing Selmer groups}

We continue to fix a number field $K$, an elliptic curve $E / K$, and a quadratic extension $F / K$.

Definition 3.1 If $T$ is a finite set of places of $K$, let

$$
\operatorname{loc}_{T}: H^{1}(K, E[2]) \longrightarrow \bigoplus_{v \in T} H^{1}\left(K_{v}, E[2]\right)
$$

denote the sum of the localization maps. Define strict and relaxed 2-Selmer groups $\mathcal{S}_{T} \subset \mathcal{S}^{T} \subset H^{1}(K, E[2])$ by the exactness of

$0 \longrightarrow \mathcal{S}^{T} \longrightarrow H^{1}(K, E[2]) \longrightarrow \bigoplus_{v \notin T} H^{1}\left(K_{v}, E[2]\right) / H_{\mathrm{f}}^{1}\left(K_{v}, E[2]\right)$,

$0 \longrightarrow \mathcal{S}_{T} \longrightarrow \mathcal{S}^{T} \longrightarrow \operatorname{loc}_{T} \longrightarrow \bigoplus_{v \in T} H^{1}\left(K_{v}, E[2]\right)$. 
Then by definition $\mathcal{S}_{T} \subset \operatorname{Sel}_{2}(E / K) \subset \mathcal{S}^{T}$, and we define

$$
V_{T}:=\operatorname{loc}_{T}\left(\operatorname{Sel}_{2}(E / K)\right) \subset \bigoplus_{v \in T} H_{\mathrm{f}}^{1}\left(K_{v}, E[2]\right) .
$$

Lemma 3.2 $\operatorname{dim}_{\mathbf{F}_{2}} \mathcal{S}^{T}-\operatorname{dim}_{\mathbf{F}_{2}} \mathcal{S}_{T}=\sum_{v \in T} \operatorname{dim}_{\mathbf{F}_{2}} H_{\mathrm{f}}^{1}\left(K_{v}, E[2]\right)$.

Proof We have exact sequences

$$
\begin{aligned}
& 0 \longrightarrow \operatorname{Sel}_{2}(E / K) \longrightarrow \mathcal{S}^{T} \stackrel{\operatorname{loc}_{T}}{\longrightarrow} \bigoplus_{v \in T}\left(H^{1}\left(K_{v}, E[2]\right) / H_{\mathrm{f}}^{1}\left(K_{v}, E[2]\right)\right) \\
& 0 \longrightarrow \mathcal{S}_{T} \longrightarrow \operatorname{Sel}_{2}(E / K) \stackrel{\operatorname{loc}_{T}}{\longrightarrow} \bigoplus_{v \in T} H_{\mathrm{f}}^{1}\left(K_{v}, E[2]\right) .
\end{aligned}
$$

By Poitou-Tate global duality (see for example [17, Theorem I.4.10], [28, Theorem 3.1], or [22, Theorem 1.7.3]), the images of the right-hand maps are orthogonal complements under the (nondegenerate) sum of the local Tate pairings, so their $\mathbf{F}_{2}$-dimensions sum to $\sum_{v \in T} \operatorname{dim}_{\mathbf{F}_{2}} H_{\mathrm{f}}^{1}\left(K_{v}, E[2]\right)$. The lemma follows directly.

Proposition 3.3 Suppose that all of the following places split in $F / K$ :

- all primes where $E$ has additive reduction,

- all $v$ of multiplicative reduction such that $\operatorname{ord}_{v}\left(\Delta_{E}\right)$ is even,

- all primes above 2 ,

- all real places $v$ with $\left(\Delta_{E}\right)_{v}>0$,

and suppose in addition that all $v$ of multiplicative reduction such that $\operatorname{ord}_{v}\left(\Delta_{E}\right)$ is odd are unramified in $F / K$.

Let $T$ be the set of (finite) primes $\mathfrak{p}$ of $K$ such that $F / K$ is ramified at $\mathfrak{p}$ and $E\left(K_{\mathfrak{p}}\right)[2] \neq 0$. Then

$$
d_{2}\left(E^{F} / K\right)=d_{2}(E / K)-\operatorname{dim}_{\mathbf{F}_{2}} V_{T}+d
$$

for some d satisfying

$$
\begin{aligned}
& 0 \leq d \leq \operatorname{dim}_{\mathbf{F}_{2}}\left(\bigoplus_{\mathfrak{p} \in T} H_{\mathrm{f}}^{1}\left(K_{\mathfrak{p}}, E[2]\right) / V_{T}\right), \\
& d \equiv \operatorname{dim}_{\mathbf{F}_{2}}\left(\bigoplus_{\mathfrak{p} \in T} H_{\mathrm{f}}^{1}\left(K_{\mathfrak{p}}, E[2]\right) / V_{T}\right)(\bmod 2) .
\end{aligned}
$$

Proof Let $V_{T}^{F}:=\operatorname{loc}_{T}\left(\operatorname{Sel}_{2}\left(E^{F} / K\right)\right) \subset \bigoplus_{\mathfrak{p} \in T} H_{\mathrm{f}}^{1}\left(K_{\mathfrak{p}}, E[2]\right)$. 
By Lemma 2.10, $H_{\mathrm{f}}^{1}\left(K_{v}, E[2]\right)=H_{\mathrm{f}}^{1}\left(K_{v}, E^{F}[2]\right)$ if $v \notin T$. Therefore we have $\mathcal{S}_{T} \subset \operatorname{Sel}_{2}\left(E^{F} / K\right) \subset \mathcal{S}^{T}$, and we have exact sequences

$$
\begin{aligned}
& 0 \longrightarrow \mathcal{S}_{T} \longrightarrow \operatorname{Sel}_{2}(E / K) \stackrel{\operatorname{loc}_{T}}{\longrightarrow} V_{T} \longrightarrow 0 \\
& 0 \longrightarrow \mathcal{S}_{T} \longrightarrow \operatorname{Sel}_{2}\left(E^{F} / K\right) \stackrel{\operatorname{loc}_{T}}{\longrightarrow} V_{T}^{F} \longrightarrow 0 .
\end{aligned}
$$

We deduce that

$$
d_{2}\left(E^{F} / K\right)=d_{2}(E / K)+\operatorname{dim}_{\mathbf{F}_{2}} V_{T}^{F}-\operatorname{dim}_{\mathbf{F}_{2}} V_{T} .
$$

Let

$$
t:=\sum_{\mathfrak{p} \in T} \operatorname{dim}_{\mathbf{F}_{2}} H_{\mathrm{f}}^{1}\left(K_{\mathfrak{p}}, E[2]\right)
$$

By Lemma 2.11 we have $\operatorname{Sel}_{2}(E / K) \cap \operatorname{Sel}_{2}\left(E^{F} / K\right)=\mathcal{S}_{T}$, and by the remark above we also have $\operatorname{Sel}_{2}(E / K)+\operatorname{Sel}_{2}\left(E^{F} / K\right) \subset \mathcal{S}^{T}$. Hence

$$
\begin{aligned}
\operatorname{dim}_{\mathbf{F}_{2}} V_{T}+\operatorname{dim}_{\mathbf{F}_{2}} V_{T}^{F} & =\operatorname{dim}_{\mathbf{F}_{2}}\left(\operatorname{Sel}_{2}(E / K) / \mathcal{S}_{T}\right)+\operatorname{dim}_{\mathbf{F}_{2}}\left(\operatorname{Sel}_{2}\left(E^{F} / K\right) / \mathcal{S}_{T}\right) \\
& \leq \operatorname{dim}_{\mathbf{F}_{2}}\left(\mathcal{S}^{T} / \mathcal{S}_{T}\right)=t
\end{aligned}
$$

where the final equality holds by Lemma 3.2.

Recall the local norm index $\delta_{v}(E, F / K)$ of Definition 2.6. By Lemma 2.10, $\delta_{v}(E, F / K)=0$ if $v \notin T$, and by Lemma 2.11,

$$
\sum_{\mathfrak{p} \in T} \delta_{v}(E, F / K)=t
$$

so $d_{2}\left(E^{F} / K\right) \equiv d_{2}(E / K)+t(\bmod 2)$ by Kramer's congruence (Theorem 2.7). Comparing this with (3) we see that

$$
\operatorname{dim}_{\mathbf{F}_{2}} V_{T}^{F} \equiv t-\operatorname{dim}_{\mathbf{F}_{2}} V_{T}=\operatorname{dim}_{\mathbf{F}_{2}}\left(\bigoplus_{\mathfrak{p} \in T} H_{\mathrm{f}}^{1}\left(K_{\mathfrak{p}}, E[2]\right) / V_{T}\right)(\bmod 2) .
$$

By (4) we have

$$
0 \leq \operatorname{dim}_{\mathbf{F}_{2}} V_{T}^{F} \leq t-\operatorname{dim}_{\mathbf{F}_{2}} V_{T}=\operatorname{dim}_{\mathbf{F}_{2}}\left(\bigoplus_{\mathfrak{p} \in T} H_{\mathrm{f}}^{1}\left(K_{\mathfrak{p}}, E[2]\right) / V_{T}\right) .
$$

If we let $d=\operatorname{dim}_{\mathbf{F}_{2}} V_{T}^{F}$, then the conclusion of the proposition follows from (3), (5), and (6). 
Corollary 3.4 Suppose E, $F / K$, and $T$ are as in Proposition 3.3.

(i) If $\operatorname{dim}_{\mathbf{F}_{2}}\left(\bigoplus_{\mathfrak{p} \in T} H_{\mathrm{f}}^{1}\left(K_{\mathfrak{p}}, E[2]\right) / V_{T}\right) \leq 1$, then

$$
d_{2}\left(E^{F} / K\right)=d_{2}(E / K)-2 \operatorname{dim}_{\mathbf{F}_{2}} V_{T}+\sum_{\mathfrak{p} \in T} \operatorname{dim}_{\mathbf{F}_{2}} H_{\mathrm{f}}^{1}\left(K_{\mathfrak{p}}, E[2]\right) .
$$

(ii) If $E\left(K_{\mathfrak{p}}\right)[2]=0$ for every $\mathfrak{p} \in T$, then $d_{2}\left(E^{F} / K\right)=d_{2}(E / K)$.

Proof The first assertion follows directly from Proposition 3.3. For (ii), note that $T$ is empty in this case, so (ii) follows from (i).

Let $M:=K(E[2])$. If $c \in H^{1}(K, E[2])$ and $\sigma \in G_{K}$, let

$$
c(\sigma) \in E[2] /(\sigma-1) E[2]
$$

denote the image of $\sigma$ under any cocycle representing $c$. This is well-defined.

Lemma 3.5 Suppose $\operatorname{Gal}(M / K) \cong S_{3}$ and $\sigma \in G_{K}$. Suppose that $C$ is a finite subgroup of $H^{1}(K, E[2])$, and $\phi: C \rightarrow E[2] /(\sigma-1) E[2]$ is a homomorphism.

Then there is a $\gamma \in G_{K}$ such that $\left.\gamma\right|_{M K^{\mathrm{ab}}}=\left.\sigma\right|_{M K^{\mathrm{ab}}}$ and $c(\gamma)=\phi(c)$ for all $c \in C$.

Proof Let $\Gamma:=\operatorname{Gal}(M / K) \cong \operatorname{Aut}(E[2])$. Then $H^{1}(\Gamma, E[2])=0$, so the restriction map

$$
H^{1}(K, E[2]) \hookrightarrow \operatorname{Hom}\left(G_{M}, E[2]\right)^{\Gamma}
$$

is injective.

Fix cocycles $\left\{c_{1}, \ldots, c_{k}\right\}$ representing an $\mathbf{F}_{2}$-basis of $C$. Then $c_{1}, \ldots, c_{k}$ restrict to linearly independent homomorphisms $\tilde{c}_{1}, \ldots, \tilde{c}_{k} \in \operatorname{Hom}\left(G_{M}, E[2]\right)^{\Gamma}$.

Let $N \subset \bar{K}$ be the (abelian) extension of $M$ fixed by $\bigcap_{i} \operatorname{ker}\left(\tilde{c}_{i}\right) \subset G_{M}$. Put $W:=G_{M} / \bigcap_{i} \operatorname{ker}\left(\tilde{c}_{i}\right)=\operatorname{Gal}(N / M)$. Then $W$ is an $\mathbf{F}_{2}$-vector space with an action of $\Gamma, \tilde{c}_{1}, \ldots, \tilde{c}_{k}$ are linearly independent in $\operatorname{Hom}(W, E[2])^{\Gamma}$, and

$$
\tilde{c}_{1} \times \cdots \times \tilde{c}_{k}: W \hookrightarrow E[2]^{k}
$$

is a $\Gamma$-equivariant injection. Thus $W$ is isomorphic to a $\Gamma$-submodule of the semisimple module $E[2]^{k}$, so $W$ is also semisimple. But if $U$ is an irreducible constituent of $W$, then $U$ is also an irreducible constituent of $E[2]^{k}$, so $U \cong E[2]$. Therefore $W \cong E[2]^{j}$ for some $j \leq k$. But then $j=$ $\operatorname{dim}_{\mathbf{F}_{2}} \operatorname{Hom}(W, E[2])^{\Gamma} \geq k$, so $j=k$ and (7) is an isomorphism.

The group $\Gamma$ acts trivially on $\operatorname{Gal}\left(\left(M K^{\mathrm{ab}} \cap N\right) / M\right)$ by conjugation, but $\operatorname{Gal}(N / M)=W \cong E[2]^{k}$ has no nonzero quotients on which $\Gamma$ acts trivially, so $M K^{\mathrm{ab}} \cap N=M$. 
Since (7) is surjective and $M K^{\mathrm{ab}} \cap N=M$, we can choose $\tau \in G_{M}$ such that $c_{i}(\tau)=\phi\left(c_{i}\right)-c_{i}(\sigma)$ for $1 \leq i \leq k$, and $\left.\tau\right|_{M K^{\mathrm{ab}}}=1$. Then $c_{i}(\tau \sigma)=$ $c_{i}(\tau)+\tau\left(c_{i}(\sigma)\right)=\phi\left(c_{i}\right)$ for every $i$. Since the $c_{i}$ represent a basis of $C$, the proposition is satisfied with $\gamma:=\tau \sigma$.

Lemma 3.6 Suppose $E(K)[2]=0$, and $c_{1}, c_{2}$ are cocycles representing distinct nonzero elements of $H^{1}(K, E[2])$. Then there is a $\gamma \in G_{K}$ such that $\left.\gamma\right|_{M K^{\mathrm{ab}}}=1$ and $c_{1}(\gamma), c_{2}(\gamma)$ are an $\mathbf{F}_{2}$-basis of $E[2]$.

Proof Let $\Gamma:=\operatorname{Gal}(M / K)$, so either $\Gamma \cong S_{3}$ or $\Gamma \cong \mathbf{Z} / 3 \mathbf{Z}$. In either case $E[2]$ is an irreducible $\Gamma$-module, and $H^{1}(\Gamma, E[2])=0$, so the restriction map

$$
H^{1}(K, E[2]) \hookrightarrow \operatorname{Hom}\left(G_{M}, E[2]\right)^{\Gamma}
$$

is injective. Let $\tilde{c}_{1}, \tilde{c}_{2}$ be the distinct nonzero elements of $\operatorname{Hom}\left(G_{M}, E[2]\right)^{\Gamma}$ obtained by restricting $c_{1}, c_{2}$ to $G_{M}$.

For $i=1,2$ let $N_{i}$ be the fixed field of $\operatorname{ker}\left(\tilde{c}_{i}\right)$. Then $\tilde{c}_{i}: \operatorname{Gal}\left(N_{i} / M\right) \rightarrow$ $E[2]$ is nonzero and $\Gamma$-equivariant, so it must be an isomorphism.

Let $N=N_{1} \cap N_{2}$. Since $\tilde{c}_{i}$ identifies $\operatorname{Gal}\left(N_{i} / N\right)$ with a $\Gamma$-stable subgroup of $E$ [2], we either have $N_{1}=N_{2}$ or $N_{1} \cap N_{2}=M$.

If $N_{1}=N_{2}$, then $\tilde{c}_{1}, \tilde{c}_{2}: \operatorname{Gal}(N / M) \rightarrow E$ [2] are different isomorphisms, so we can find $\tau \in \operatorname{Gal}(N / M)$ such that $\tilde{c}_{1}(\tau)$ and $\tilde{c}_{2}(\tau)$ are distinct and nonzero.

If $N_{1} \cap N_{2}=M$, then again we can find $\tau \in \operatorname{Gal}\left(N_{1} N_{2} / M\right)$ such that $\tilde{c}_{1}(\tau)$ and $\tilde{c}_{2}(\tau)$ are distinct and nonzero.

Since $\Gamma$ acts trivially on $\operatorname{Gal}\left(\left(M K^{\mathrm{ab}} \cap N_{1} N_{2}\right) / M\right)$, but $\operatorname{Gal}\left(N_{1} N_{2} / M\right) \cong$ $E[2]$ or $E[2]^{2}$ has no nonzero quotients on which $\Gamma$ acts trivially, we have $M K^{\mathrm{ab}} \cap N_{1} N_{2}=M$. Thus we can choose $\gamma \in G_{M}$ such that $\left.\gamma\right|_{M K^{\mathrm{ab}}}=1$ and $\left.\gamma\right|_{N_{1} N_{2}}=\tau$. This $\gamma$ has the desired properties.

\section{Proof of Theorem 1.4}

In this section we will prove Theorem 1.4. Suppose $K$ is a number field, $N$ is a finite abelian extension of $K$, and $M$ is another Galois extension of $K$.

Fix a nonempty union of conjugacy classes $S \subset \mathrm{Gal}(M / K)$. If $\mathfrak{p}$ is a prime of $K$ unramified in $M / K$, let $\operatorname{Frob}_{\mathfrak{p}}(M / K)$ denote the Frobenius (conjugacy class) of $\mathfrak{p}$ in $\operatorname{Gal}(M / K)$. Define a set of primes of $K$

$$
\mathcal{P}:=\left\{\mathfrak{p}: \mathfrak{p} \text { is unramified in } N M / K \text { and } \operatorname{Frob}_{\mathfrak{p}}(M / K) \subset S\right\}
$$

and two sets of ideals $\mathcal{N}_{1} \subset \mathcal{N}$ of $K$ 


$$
\begin{aligned}
\mathcal{N} & :=\{\mathfrak{a}: \mathfrak{a} \text { is a squarefree product of primes in } \mathcal{P}\}, \\
\mathcal{N}_{1} & :=\{\mathfrak{a} \in \mathcal{N}:[\mathfrak{a}, N / K]=1\},
\end{aligned}
$$

where $[\cdot, N / K]$ is the global Artin symbol.

Lemma 4.1 There is a positive real constant $C$ such that

$$
\left|\left\{\mathfrak{a} \in \mathcal{N}_{1}: \mathbf{N}_{K / \mathbf{Q}} \mathfrak{a}<X\right\}\right|=(C+o(1)) \frac{X}{(\log X)^{1-|S| /[M: K]}} .
$$

Proof The proof is a straightforward adaptation of a result of Serre [23, Théorème 2.4], who proved this when $K=N=\mathbf{Q}$.

Let $G=\operatorname{Gal}(N / K)$. If $\chi: G \rightarrow \mathbf{C}^{\times}$is a character, let

$$
f_{\chi}(s):=\sum_{\mathfrak{a} \in \mathcal{N}} \chi(\mathfrak{a}) \mathbf{N a} \mathfrak{a}^{-s}=\prod_{\mathfrak{p} \in \mathcal{P}}\left(1+\chi(\mathfrak{p}) \mathbf{N p}^{-s}\right)
$$

where $\chi(\mathfrak{a})=\chi([\mathfrak{a}, N / K])$. Then standard methods show that

$$
\log f_{\chi}(s)=\sum_{\mathfrak{p} \in \mathcal{P}} \log \left(1+\chi(\mathfrak{p}) \mathbf{N p}^{-s}\right) \sim \sum_{\mathfrak{p} \in \mathcal{P}} \chi(\mathfrak{p}) \mathbf{N p}^{-s} \sim \delta_{\chi} \log \left(\frac{1}{s-1}\right)
$$

where

$$
\delta_{\chi}:= \begin{cases}0 & \text { if } \chi \text { is not the trivial character, } \\ |S| /[M: K] & \text { if } \chi \text { is trivial, }\end{cases}
$$

and we write $g(s) \sim h(s)$ for functions $g, h$ defined on the half-plane $\Re(s)>1$ to mean that $g(s)-h(s)$ extends to a holomorphic function on $\Re(s) \geq 1$. It follows that

$$
\sum_{\mathfrak{a} \in \mathcal{N}_{1}} \mathbf{N a}^{-s}=\frac{1}{[N: K]} \sum_{\chi} f_{\chi}(s)=(s-1)^{-|S| /[M: K]} g(s)
$$

with a function $g(s)$ that is holomorphic and nonzero on $\Re(s) \geq 1$. The lemma now follows from a variant of Ikehara's Tauberian Theorem [29, p. 322].

Now fix an elliptic curve $E$ over $K$ with $E[2]=0$, and let $\Delta$ be the discriminant of an integral model of $E$. Let $N=K(8 \Delta \infty)$, the ray class field of $K$ modulo $8 \Delta$ and all archimedean places, and let $M:=K(E[2])$. Let $\mathcal{P}$ and $\mathcal{N}_{1}$ be as defined above, with this $N$ and $M$ and with $S$ the set of elements of order 3 in $\operatorname{Gal}(M / K)$. Since $E(K)[2]=0$ we have $|S|=2$.

Proposition 4.2 Suppose $\mathfrak{a} \in \mathcal{N}_{1}$. Then there is a quadratic extension $F / K$ of conductor a such that $d_{2}\left(E^{F}\right)=d_{2}(E)$. 
Proof Fix $\mathfrak{a} \in \mathcal{N}_{1}$. Then $\mathfrak{a}$ is principal, with a totally positive generator $\alpha \equiv 1(\bmod 8 \Delta)$. Let $F=K(\sqrt{\alpha})$. Then all primes above 2 , all primes of bad reduction, and all infinite places split in $F / K$. If $\mathfrak{p}$ ramifies in $F / K$ then $\mathfrak{p} \mid \mathfrak{a}$, so $\mathfrak{p} \in \mathcal{P}$. Thus the Frobenius of $\mathfrak{p}$ in $\operatorname{Gal}(M / K)$ has order 3, which shows that $E\left(K_{\mathfrak{p}}\right)[2]=0$. Now the proposition follows from Corollary 3.4(ii).

Proof of Theorem 1.4 Recall that $S$ is the set of elements of order 3 in $\operatorname{Gal}(M / K)$, so

$$
\frac{|S|}{[M: K]}= \begin{cases}1 / 3 & \text { if } \operatorname{Gal}(M / K) \cong S_{3}, \\ 2 / 3 & \text { if } \operatorname{Gal}(M / K) \cong \mathbf{Z} / 3 \mathbf{Z}\end{cases}
$$

Case 1: $d_{2}(E / K)=r$. By Proposition 4.2,

$$
N_{r}(E, X) \geq\left|\left\{\mathfrak{a} \in \mathcal{N}_{1}: \mathbf{N}_{K / \mathbf{Q}} \mathfrak{a}<X\right\}\right| .
$$

The estimate of Lemma 4.1 for the right-hand side of this inequality proves Theorem 1.4 in this case.

Case 2: $d_{2}(E / K)$ arbitrary. We have assumed that $E$ has a twist $E^{L}$ with $d_{2}\left(E^{L} / K\right)=r$. Every twist $\left(E^{L}\right)^{F^{\prime}}$ of $E^{L}$ is also a twist $E^{F}$ of $E$, and

$$
\mathfrak{f}(F / K) \mid \mathfrak{f}(L / K) \mathfrak{f}\left(F^{\prime} / K\right)
$$

so $N_{r}(E, X) \geq N_{r}\left(E^{L}, X / \mathbf{N}_{K / \mathbf{Q}} \mathfrak{f}(L / K)\right)$. Now Theorem 1.4 for $E$ follows from Theorem 1.4 for $E^{L}$, which is proved in Case 1.

\section{Twisting to lower and raise the Selmer rank}

In this section we will use Corollary 3.4 and Lemmas 3.5 and 3.6 to prove Theorems 1.5, 1.6, and 1.7:

(1) Lemmas 3.5 or 3.6 will provide us with Galois automorphisms that evaluate Selmer cocycles in some useful way,

(2) the Cebotarev Theorem will provide us with primes whose Frobenius automorphisms are the Galois automorphisms we chose in (1),

(3) Corollary 3.4 will enable us to calculate $d_{2}\left(E^{F} / K\right)$, where $F$ is a quadratic extension ramified at one of the primes chosen in (2).

We use Proposition 5.1 below to prove Theorem 1.6, Proposition 5.2 to prove Theorem 1.7, and Proposition 5.3 to prove Theorem 1.5. We also prove Corollaries $1.8,1.9,1.10,1.11$, and 1.12. 
Proposition 5.1 Suppose E/K satisfies the hypotheses of Theorem 1.6. Suppose $L / K$ is a quadratic extension (or $L=K$ ) such that the place $v_{0}$ of Theorem 1.6 is unramified in $L / K, L^{\prime} / K$ is a cyclic extension of odd degree, and $\Sigma$ is a finite set of places of $K$.

(i) There is a quadratic twist $A$ of $E$ such that $d_{2}(A / K)=d_{2}(E / K)+1$ and $d_{2}\left(A^{L} / K\right)=d_{2}\left(E^{L} / K\right)+1$.

(ii) If $d_{2}(E / K)>0$ and $d_{2}\left(E^{L} / K\right)>0$, then there is a quadratic twist $A$ of $E$ such that $d_{2}(A / K)=d_{2}(E / K)-1$ and $d_{2}\left(A^{L} / K\right)=d_{2}\left(E^{L} / K\right)-1$.

(iii) If $\operatorname{Sel}_{2}\left(E^{L} / K\right) \not \subset \operatorname{Sel}_{2}(E / K)$ inside $H^{1}(K, E[2])$, then there is a quadratic twist $A$ of $E$ such that $d_{2}(A / K)=d_{2}(E / K)+1$ and $d_{2}\left(A^{L} / K\right)=d_{2}\left(E^{L} / K\right)-1$.

In all three cases we can take $A=E^{F}$, where the quadratic extension $F / K$ satisfies:

- all places in $\Sigma-\left\{v_{0}\right\}$ split in $F / K$,

- $F / K$ ramifies at exactly one prime $\mathfrak{p}$, and that prime satisfies $\mathfrak{p} \notin \Sigma, \mathfrak{p}$ is inert in $L^{\prime}$, and $E\left(K_{\mathfrak{p}}\right)[2] \cong \mathbf{Z} / 2 \mathbf{Z}$.

Proof Let $\Delta$ be the discriminant of (some integral model of) $E$. Let $M:=$ $K(E[2])=K\left(E^{L}[2]\right)$, so $M$ is an $S_{3}$-extension of $K$ containing the quadratic extension $K(\sqrt{\Delta})$. Enlarge $\Sigma$ if necessary so that it includes all infinite places, all primes above 2 , and all primes where either $E$ or $E^{L}$ has bad reduction. Let $v_{0} \nmid 2$ be the distinguished place of Theorem 1.6, either real with $\Delta_{v_{0}}<0$, or of multiplicative reduction with $\operatorname{ord}_{v_{0}}(\Delta)$ odd.

Let $\mathfrak{d}$ be the (formal) product of all places in $\Sigma-\left\{v_{0}\right\}$. Let $K(8 \mathfrak{d})$ denote the ray class field of $K$ modulo $8 \mathfrak{d}$, and let $K[8 \mathfrak{d}]$ denote the maximal 2power extension of $K$ in $K(8 \mathfrak{d})$. Note that $K(\sqrt{\Delta}) / K$ is ramified at $v_{0}$ but $K[8 \mathfrak{d}] / K$ is not, and $\left[L^{\prime}: K\right]$ is odd, so the fields $K[8 \mathfrak{d}], L^{\prime}, M$ are linearly disjoint. Therefore we can fix an element $\sigma \in G_{K}$ such that

- $\left.\sigma\right|_{M} \in \operatorname{Gal}(M / K) \cong S_{3}$ has order 2 ,

- $\left.\sigma\right|_{K[80]}=1$,

- $\left.\sigma\right|_{L^{\prime}}$ is a generator of $\operatorname{Gal}\left(L^{\prime} / K\right)$.

It follows in particular that $E[2] /(\sigma-1) E[2] \cong \mathbf{Z} / 2 \mathbf{Z}$.

Let $C=\operatorname{Sel}_{2}(E / K)+\operatorname{Sel}_{2}\left(E^{L} / K\right) \subset H^{1}(K, E[2])$, and suppose $\phi: C \rightarrow$ $E[2] /(\sigma-1) E[2]$ is a homomorphism. By Lemma 3.5 we can find $\gamma \in G_{K}$ such that

- $\left.\gamma\right|_{M L^{\prime} K[80]}=\sigma$,

- $c(\gamma)=\phi(c)$ for every $c \in C$.

Let $N$ be a Galois extension of $K$ containing $M L^{\prime} K[8 \mathfrak{d}]$, large enough so that the restriction of $C$ to $N$ is zero. (For example, one can take the 
compositum of $L^{\prime} K(8 \mathfrak{d})$ with the fixed field of the intersection of the kernels of the restrictions of $c \in C \hookrightarrow \operatorname{Hom}\left(G_{M}, E[2]\right)$.) Let $\mathfrak{p}$ be a prime of $K$ not in $\Sigma$, whose Frobenius in $\operatorname{Gal}(N / K)$ is the conjugacy class of $\gamma$. Since $\left.\gamma\right|_{K[8 \mathfrak{d}]}=\left.\sigma\right|_{K[8 \mathfrak{d}]}=1$, and $[K(8 \mathfrak{d}): K[8 \mathfrak{d}]]$ is odd, there is an odd positive integer $h$ such that $\left.\gamma^{h}\right|_{K(80)}=1$. Thus $\mathfrak{p}^{h}$ is principal, with a generator $\pi \equiv 1$ $(\bmod 8 \mathfrak{d})$, positive at all real embeddings different from $v_{0}$. Let $F=K(\sqrt{\pi})$. Then all places $v \in \Sigma-\left\{v_{0}\right\}$ split in $F, F / K$ is ramified at $\mathfrak{p}$ and nowhere else, $\mathfrak{p}$ is inert in $L^{\prime} / K$ because $\left.\gamma\right|_{L^{\prime}}$ generates $\operatorname{Gal}\left(L^{\prime} / K\right)$, and $E\left(K_{\mathfrak{p}}\right)[2] \neq 0$ because Frob $\left.\right|_{\mathfrak{p}} L_{[2]}=\left.\sigma\right|_{E[2]}$ has order 2 .

We will apply Corollary 3.4, with $T=\{\mathfrak{p}\}$. Since $E$ has good reduction at $\mathfrak{p}$, it follows from Lemma 2.2(ii) that

$$
H_{\mathrm{f}}^{1}\left(K_{\mathfrak{p}}, E[2]\right) \cong E[2] /\left(\text { Frob }_{\mathfrak{p}}-1\right) E[2]=E[2] /(\sigma-1) E[2],
$$

and similarly with $E$ replaced by $E^{L}$, so

$$
\operatorname{dim}_{\mathbf{F}_{2}} H_{\mathrm{f}}^{1}\left(K_{\mathfrak{p}}, E[2]\right)=\operatorname{dim}_{\mathbf{F}_{2}} H_{\mathrm{f}}^{1}\left(K_{\mathfrak{p}}, E^{L}[2]\right)=1 .
$$

Further, the localization maps

$$
\operatorname{loc}_{T}: \operatorname{Sel}_{2}(E / K), \operatorname{Sel}_{2}\left(E^{L} / K\right) \longrightarrow H_{\mathrm{f}}^{1}\left(K_{\mathfrak{p}}, E[2]\right) \stackrel{\sim}{\rightarrow} E[2] /(\sigma-1) E[2]
$$

are given by evaluation of cocycles at Frob $b_{\mathfrak{p}}=\gamma$. Hence by our choice of $\gamma$, (8) identifies

$$
\begin{aligned}
& \operatorname{loc}_{T}\left(\operatorname{Sel}_{2}(E / K)\right)=\phi\left(\operatorname{Sel}_{2}(E / K)\right), \\
& \operatorname{loc}_{T}\left(\operatorname{Sel}_{2}\left(E^{L} / K\right)\right)=\phi\left(\operatorname{Sel}_{2}\left(E^{L} / K\right)\right) .
\end{aligned}
$$

Thus by Corollary 3.4(i) we conclude that

$$
\begin{aligned}
d_{2}\left(E^{F} / K\right) & = \begin{cases}d_{2}(E / K)+1 & \text { if } \phi\left(\operatorname{Sel}_{2}(E / K)\right)=0, \\
d_{2}(E / K)-1 & \text { if } \phi\left(\operatorname{Sel}_{2}(E / K)\right) \neq 0,\end{cases} \\
d_{2}\left(\left(E^{F}\right)^{L} / K\right) & =d_{2}\left(\left(E^{L}\right)^{F} / K\right) \\
& = \begin{cases}d_{2}\left(E^{L} / K\right)+1 & \text { if } \phi\left(\operatorname{Sel}_{2}\left(E^{L} / K\right)\right)=0, \\
d_{2}\left(E^{L} / K\right)-1 & \text { if } \phi\left(\operatorname{Sel}_{2}\left(E^{L} / K\right)\right) \neq 0 .\end{cases}
\end{aligned}
$$

For assertion (i), let $\phi=0$. For (ii), if $d_{2}(E / K)>0$ and $d_{2}\left(E^{L} / K\right)>0$, then we can choose a $\phi$ that is nonzero on both $\operatorname{Sel}_{2}(E / K)$ and $\operatorname{Sel}_{2}\left(E^{L} / K\right)$. For (iii), if $\operatorname{Sel}_{2}\left(E^{L} / K\right) \not \subset \operatorname{Sel}_{2}(E / K)$, then we can choose a $\phi$ that is zero on $\operatorname{Sel}_{2}(E / K)$ and nonzero on $\operatorname{Sel}_{2}\left(E^{L} / K\right)$. In all three cases, the proposition holds with $A=E^{F}$. 
Proof of Theorem 1.6 Note that if $E$ satisfies the hypotheses of Theorem 1.6, then so does every quadratic twist of $E$.

If $r \geq d_{2}(E / K)$, then applying Proposition 5.1(i) $r-d_{2}(E / K)$ times (with $\left.L=L^{\prime}=K\right)$ shows that $E$ has a twist $E^{\prime}$ with $d_{2}\left(E^{\prime} / K\right)=r$.

If $0 \leq r \leq d_{2}(E / K)$ then applying Proposition 5.1(ii) $d_{2}(E / K)-r$ times shows that $E$ has a twist $E^{\prime}$ with $d_{2}\left(E^{\prime} / K\right)=r$.

Now Theorem 1.4 shows that for every $r \geq 0, E$ has many twists $E^{\prime}$ with $d_{2}\left(E^{\prime} / K\right)=r$.

Proposition 5.2 Suppose $E / K$ is an elliptic curve such that $E(K)[2]$ $=0$. If $d_{2}(E / K)>1$, then $E$ has a quadratic twist $E^{F}$ over $K$ such that $d_{2}\left(E^{F} / K\right)=d_{2}(E / K)-2$.

Proof The proof is similar to that of Proposition 5.1(ii). Let $M:=K(E[2])$, and let $\Delta$ be the discriminant of (some integral model of) $E$. Let $K(8 \Delta \infty)$ denote the ray class field of $K$ modulo the product of $8 \Delta$ and all infinite places.

Since $d_{2}(E / K)>1$, we can choose cocycles $c_{1}, c_{2}$ representing $\mathbf{F}_{2}$ independent elements of $\operatorname{Sel}_{2}(E / K)$. By Lemma 3.6 we can find $\gamma \in G_{K}$ such that

- $\left.\gamma\right|_{M K(8 \Delta \infty)}=1$,

- $c_{1}(\gamma), c_{2}(\gamma)$ are an $\mathbf{F}_{2}$-basis of $E[2]$.

Let $N$ be a Galois extension of $K$ containing $M K(8 \Delta \infty)$, large enough so that the restriction of $\operatorname{Sel}_{2}(E / K)$ to $N$ is zero. Let $\mathfrak{p}$ be a prime of $K$ where $E$ has good reduction, not dividing 2, whose Frobenius in $\operatorname{Gal}(N / K)$ is the conjugacy class of $\gamma$. Then $\mathfrak{p}$ has a totally positive generator $\pi \equiv 1$ $(\bmod 8 \Delta)$. Let $F=K(\sqrt{\pi})$. Then all places $v$ dividing $2 \Delta \infty$ split in $F / K$, and $\mathfrak{p}$ is the only prime that ramifies in $F / K$.

We will apply Corollary 3.4 with $T=\{\mathfrak{p}\}$. Since $E$ has good reduction at $\mathfrak{p}$, it follows from Lemma 2.2(ii) that

$$
H_{\mathrm{f}}^{1}\left(K_{\mathfrak{p}}, E[2]\right)=E[2] /\left(\text { Frob }_{\mathfrak{p}}-1\right) E[2]=E[2] /(\gamma-1) E[2]=E[2] .
$$

The localization map $\operatorname{loc}_{T}: \operatorname{Sel}_{2}(E / K) \rightarrow H_{\mathrm{f}}^{1}\left(K_{\mathfrak{p}}, E[2]\right)$ is given by evaluation of cocycles at Frob $b_{\mathfrak{p}}=\gamma$, so by our choice of $\gamma$, the classes $\operatorname{loc}_{T}\left(c_{1}\right)$ and $\operatorname{loc}_{T}\left(c_{2}\right)$ generate $H_{\mathrm{f}}^{1}\left(K_{\mathfrak{p}}, E[2]\right)$. In particular $\operatorname{loc}_{T}$ is surjective, so in the notation of Corollary 3.4 we have $\operatorname{dim}_{\mathbf{F}_{2}} V_{T}=\operatorname{dim}_{\mathbf{F}_{2}} H_{\mathrm{f}}^{1}\left(K_{\mathfrak{p}}, E[2]\right)=2$. Corollary 3.4(i) now yields $d_{2}\left(E^{F} / K\right)=d_{2}(E / K)-2$, as desired.

Proof of Theorem 1.7 Suppose $0 \leq r \leq d_{2}(E / K)$. Applying Proposition 5.2 $\left(d_{2}(E / K)-r\right) / 2$ times shows that $E$ has a twist $E^{\prime}$ with $d_{2}\left(E^{\prime} / K\right)=r$, and then Theorem 1.4 shows that $E$ has many such twists. 
Proof of Corollary 1.8 Let $k=\mathbf{Q}(j(E)) \subset K$. Fix an elliptic curve $E_{0}$ over $k$ with $j\left(E_{0}\right)=j(E)$. Since $j(E) \neq 0,1728, E_{0}$ is a quadratic twist of $E$ over $K$. Thus $\left[k\left(E_{0}[2]\right): k\right] \geq\left[K\left(E_{0}[2]\right): K\right]=[K(E[2]): K]$, so $\operatorname{Gal}\left(k\left(E_{0}[2]\right) / k\right) \cong S_{3}$. Also

$$
j(E)-1728=j\left(E_{0}\right)-1728=c_{6}\left(E_{0}\right)^{2} / \Delta_{E_{0}}
$$

so $\left(\Delta_{E_{0}}\right)_{v}<0$ at the real embedding $v$ of $k$. Therefore $E_{0} / k$ satisfies the hypotheses of Theorem 1.6, so Theorem 1.6 shows that $d_{2}\left(E_{0}^{F} / k\right)$ can be arbitrarily large as $F$ varies through quadratic extensions of $k$. Since $E(K)[2]=0$, the map $\operatorname{Sel}_{2}\left(E_{0}^{F} / k\right) \rightarrow \operatorname{Sel}_{2}\left(E_{0}^{F} / K\right)$ is injective, and so $d_{2}\left(E^{F} / K\right)$ can be arbitrarily large as $F$ varies through quadratic extensions of $K$. Now the corollary follows from Theorem 1.7.

Proposition 5.3 Suppose $E / K$ is an elliptic curve such that $E(K)[2]=0$, and either $K$ has a real embedding, or $E$ has multiplicative reduction at some prime of $K$. Then $E$ has a quadratic twist $E^{F} / K$ such that $d_{2}\left(E^{F} / K\right) \not \equiv$ $d_{2}(E / K)(\bmod 2)$ and $d_{2}\left(E^{F} / K\right) \geq d_{2}(E / K)-1$.

Proof Let $M:=K(E[2])$, and let $\Delta$ be the discriminant of (some integral model of) $E$. Let $\mathfrak{d}$ be the (formal) product of $\Delta$ and all infinite places, let $K(8 \mathfrak{d})$ denote the ray class field of $K$ modulo $8 \mathfrak{d}$, and let $K[8 \mathfrak{d}]$ denote the maximal 2-power extension of $K$ in $K(8 \mathfrak{d})$. We have $M \cap K[8 \mathfrak{d}]=K(\sqrt{\Delta})$.

Let $v_{0}$ be the distinguished place, either real or of multiplicative reduction. Let $\mathbf{x}=\left(x_{v}\right)$ be an idele of $K$ defined by:

- $x_{v}=1$ if $v \neq v_{0}$,

- $x_{v_{0}}=-1$ if $v_{0}$ is real, $x_{v_{0}}$ is a unit at $v_{0}$ such that $K_{v_{0}}\left(\sqrt{x_{v_{0}}}\right)$ is the unramified quadratic extension of $K_{v_{0}}$ if $v_{0}$ is nonarchimedean.

Let $\sigma=[\mathbf{x}, K[8 \mathfrak{d}] / K] \in \operatorname{Gal}(K[8 \mathfrak{d}] / K)$ be the image of $\mathbf{x}$ under the global Artin map. We consider two cases.

Case 1: $\sigma(\sqrt{\Delta})=\sqrt{\Delta}$. In this case we can choose $\gamma \in \operatorname{Gal}(M K[8 \mathfrak{d}] / K)$ such that $\left.\gamma\right|_{K[80]}=\sigma$ and $\left.\gamma\right|_{M}$ has order 3 .

Case 2: $\sigma(\sqrt{\Delta})=-\sqrt{\Delta}$. In this case $\operatorname{Gal}(M / K) \cong S_{3}$, and $\sigma$ is nontrivial on $M \cap K[8 \mathfrak{d}]=K(\sqrt{\Delta})$. By Lemma 3.5 we can find $\gamma \in G_{K}$ such that $\left.\gamma\right|_{K[80]}=\sigma,\left.\gamma\right|_{M}$ has order 2, and $c(\gamma) \in(\gamma-1) E$ [2] for every cocycle $c$ representing an element of $\operatorname{Sel}_{2}(E / K)$.

In either case, let $\mathfrak{p}$ be a prime of $K$ not dividing $2 \Delta$, whose Frobenius in $\operatorname{Gal}(M K[8 \mathfrak{d}] / K)$ is $\gamma$. Then some odd power $\mathfrak{p}^{h}$ is principal, with a generator $\pi$ such that $\pi \in\left(K_{v}^{\times}\right)^{2}$ if $v \mid 2 \Delta \infty$ and $v \neq v_{0}, K_{v_{0}}(\sqrt{\pi})=\mathbf{C}$ if $v_{0}$ 
is real, and $K_{v_{0}}(\sqrt{\pi})$ is the unramified quadratic extension of $K_{v_{0}}$ if $v_{0}$ is nonarchimedean.

Let $F=K(\sqrt{\pi})$, and recall the local norm index $\delta_{v}(E, F / K)$ of Definition 2.6. All places $v \mid 2 \Delta \infty$ different from $v_{0}$ split in $F / K$, so by Lemma 2.10, $\delta_{v}(E, F / K)=0$ and $H_{\mathrm{f}}^{1}\left(K_{v}, E[2]\right)=H_{\mathrm{f}}^{1}\left(K_{v}, E^{F}[2]\right)$ if $v \neq$ $v_{0}, \mathfrak{p}$. It follows (using Kramer's congruence Theorem 2.7 for (9)) that

$$
d_{2}\left(E^{F} / K\right) \equiv d_{2}(E / K)+\delta_{v_{0}}(E, F / K)+\delta_{\mathfrak{p}}(E, F / K)(\bmod 2),
$$

and

$\operatorname{ker}\left[\operatorname{Sel}_{2}(E / K) \longrightarrow H_{\mathrm{f}}^{1}\left(K_{v_{0}}, E[2]\right) \oplus H_{\mathrm{f}}^{1}\left(K_{\mathfrak{p}}, E[2]\right)\right] \subset \operatorname{Sel}_{2}\left(E^{F} / K\right)$.

Consider the Hilbert symbol $(\Delta, \pi)_{v}$, which is 1 if $\Delta$ is a norm from $\left(F \otimes K_{v}\right)^{\times}$to $K_{v}^{\times}$, and -1 if not. Then $(\Delta, \pi)_{v}=1$ if $v \neq v_{0}, \mathfrak{p}$, and $\prod_{v}(\Delta, \pi)_{v}=1$, so $(\Delta, \pi)_{v_{0}}=(\Delta, \pi)_{\mathfrak{p}}$. By [11, Proposition 6] if $v_{0}$ is real, and by [11, Propositions 1,2$]$ if $v_{0}$ is multiplicative, we have

$$
\delta_{v_{0}}(E, F / K)= \begin{cases}1 & \text { if }(\Delta, \pi)_{v_{0}}=1, \\ 0 & \text { if }(\Delta, \pi)_{v_{0}}=-1 .\end{cases}
$$

By [11, Proposition 3], and using that $\gamma$ acts nontrivially on $E$ [2] in both Case 1 and Case 2, we have

$$
\delta_{\mathfrak{p}}(E, F / K)= \begin{cases}0 & \text { if }(\Delta, \pi)_{\mathfrak{p}}=1 \\ 1 & \text { if }(\Delta, \pi)_{\mathfrak{p}}=-1\end{cases}
$$

Thus $\delta_{v_{0}}(E, F / K)+\delta_{\mathfrak{p}}(E, F / K)=1$, so (9) shows that $d_{2}\left(E^{F} / K\right)$ and $d_{2}(E / K)$ have opposite parity.

In Case $1, E[2] /(\gamma-1) E[2]=0$, so $H_{\mathrm{f}}^{1}\left(K_{\mathfrak{p}}, E[2]\right)=0$ by Lemma 2.2(ii). In Case 2, the restriction map

$$
\operatorname{Sel}_{2}(E / K) \rightarrow H_{\mathrm{f}}^{1}\left(K_{\mathfrak{p}}, E[2]\right) \cong E[2] /(\gamma-1) E[2]
$$

is given by evaluating cocycles at $\gamma$, so by our choice of $\gamma$ this image is zero. In both cases, $\operatorname{dim}_{\mathbf{F}_{2}} H_{\mathrm{f}}^{1}\left(K_{v_{0}}, E[2]\right) \leq 2$, so by (10) we have $d_{2}\left(E^{F} / K\right) \geq$ $d_{2}(E / K)-2$. This completes the proof.

Proof of Theorem 1.5 Let $E^{F}$ be a twist of $E$ as in Proposition 5.3. Theorem 1.5 follows directly from Theorem 1.7 applied to $E$ and to $E^{F}$.

Lemma 5.4 Suppose $\mathfrak{p}$ is a prime of $K$ not dividing 2. Then there is an elliptic curve $E / K$ with all of the following properties:

(i) E is semistable at all primes,

(ii) E has multiplicative reduction at $\mathfrak{p}$ and $\operatorname{ord}_{\mathfrak{p}}\left(\Delta_{E}\right)=1$,

(iii) $\operatorname{Gal}(K(E[2]) / K) \cong S_{3}$. 
If in addition the rational prime $p$ below $\mathfrak{p}$ is unramified in the Galois closure of $K / \mathbf{Q}$, then $E$ can be taken to be the base change of an elliptic curve over $\mathbf{Q}$.

Proof Let $E_{t}$ be the elliptic curve $y^{2}+y=x^{3}-x^{2}+t$ over $K(t)$. Then

$$
\begin{aligned}
& j\left(E_{t}\right)=-\frac{2^{12}}{(4 t+1)(108 t+11)}, \quad \Delta\left(E_{t}\right)=-(4 t+1)(108 t+11), \\
& c_{4}\left(E_{t}\right)=16 .
\end{aligned}
$$

It follows from [25, Proposition VII.5.1] that for every $t \in \mathcal{O}_{K}, E_{t}$ has semistable reduction at all primes of $K$.

Let $\eta \in \mathcal{O}_{K}$ be such that $\operatorname{ord}_{\mathfrak{p}}(4 \eta+1)=1$, and let $g(t):=\eta+$ $(4 \eta+1)^{2} t$. Then for every $t \in \mathcal{O}_{K}$ we have ord $\operatorname{or}_{\mathfrak{p}}(4 g(t)+1)=1$. The splitting field of $f_{t}(x):=x^{3}-x^{2}+g(t)+1 / 4$ over $K(t)$ has Galois group $S_{3}$, since $f_{t}$ is irreducible and its discriminant $-(4 g(t)+1)(108 g(t)+11) / 16$ is not a square. Hence by Hilbert's Irreducibility Theorem, there is an integer $t_{0} \in \mathcal{O}_{K}$ such that the splitting field of $f_{t_{0}}(x)$ over $K$ is an $S_{3}$-extension.

Let $E$ be the elliptic curve $E_{g\left(t_{0}\right)}$. Then $K(E[2])$ is the splitting field of $f_{t_{0}}(x)$, so $\operatorname{Gal}(K(E[2]) / K) \cong S_{3}$, and

$$
\begin{aligned}
\Delta(E) & =-\left(4 g\left(t_{0}\right)+1\right)\left(108 g\left(t_{0}\right)+11\right) \\
& =-\left(4 g\left(t_{0}\right)+1\right)\left(27\left(4 g\left(t_{0}\right)+1\right)-16\right) .
\end{aligned}
$$

Thus $E$ satisfies (i), (ii), and (iii).

Let $K^{\prime}$ be the Galois closure of $K / \mathbf{Q}$, and $p$ the rational prime below $\mathfrak{p}$, and suppose $p$ is unramified in $K^{\prime} / \mathbf{Q}$. We can apply the lemma with $p$ and $\mathbf{Q}$ in place of $\mathfrak{p}$ and $K$ to produce a semistable elliptic curve $E / \mathbf{Q}$ such that $\operatorname{ord}_{p}\left(\Delta_{E}\right)=1$ and $\operatorname{Gal}(\mathbf{Q}(E[2]) / \mathbf{Q}) \cong S_{3}$.

Then $E / K$ satisfies (i) and (ii). Further, $\mathbf{Q}(E[2]) \cap K^{\prime}$ is a Galois extension of $\mathbf{Q}$ that does not contain $\mathbf{Q}\left(\sqrt{\Delta_{E}}\right)$ (since the latter is ramified at $p$ ). Therefore $\mathbf{Q}(E[2]) \cap K=\mathbf{Q}$, and so $\operatorname{Gal}(K(E[2]) / K) \cong \operatorname{Gal}(\mathbf{Q}(E[2]) / \mathbf{Q}) \cong S_{3}$.

Proof of Corollary 1.9 By Lemma 5.4, we can find an elliptic curve $E$ over $K$ and a prime $\mathfrak{p} \nmid 2$ such that $E$ has multiplicative reduction at $\mathfrak{p}$, $\operatorname{ord}_{\mathfrak{p}}\left(\Delta_{E}\right)=1$, and $\operatorname{Gal}(K(E[2]) / K) \cong S_{3}$. By Theorems 1.6 and 1.4, this $E$ has many quadratic twists $E^{\prime}$ with $d_{2}\left(E^{\prime} / K\right)=r$, for every $r \geq 0$.

Lemma 5.5 Suppose $E$ is an elliptic curve over $K$. Then for all but finitely many quadratic twists $E^{\prime}$ of $E, E^{\prime}(K)$ has no odd-order torsion.

Proof This is proved in [9, Proposition 1] when $K=\mathbf{Q}$; we adapt the proof given there. By Merel's Uniform Boundedness Theorem for torsion on elliptic curves [16], the set

$$
\text { \{primes } p: E^{F}(K)[p] \neq 0 \text { for some quadratic extension } F / K \text { \} }
$$


is finite. On the other hand, if $p$ is odd and $\rho_{p}: G_{K} \rightarrow \operatorname{Aut}(E[p]) \cong \mathrm{GL}_{2}\left(\mathbf{F}_{p}\right)$ denotes the mod- $p$ representation attached to $E$, then there are at most two characters $\chi$ of $G_{K}$ such that $\rho_{p} \otimes \chi$ contains a copy of the trivial representation. Therefore for fixed odd $p$, the set

$$
\left\{F / K \text { quadratic : } E^{F}(K)[p] \neq 0\right\}
$$

has order at most 2. This completes the proof.

Proof of Corollary 1.10 By Theorems 1.5 and 1.4, $E$ has many quadratic twists $E^{\prime}$ with $d_{2}\left(E^{\prime} / K\right)=0$, and hence $\operatorname{rank}\left(E^{\prime}(K)\right)=0$ by (1). Since $E(K)[2]=0$, none of these twists have rational 2-torsion, and by Lemma 5.5, only finitely many of these twists have odd-order torsion. This proves the corollary.

Proof of Corollary 1.11 (and Theorem 1.1) By Lemma 5.4 there is an elliptic curve $E$ over $K$ with multiplicative reduction at a prime $\mathfrak{p} \nmid 2$, and with $E[2]=0$. Now the Corollary 1.11 follows from Corollary 1.10.

Proof of Corollary 1.12 By Theorems 1.5 and 1.4, $E$ has many quadratic twists $E^{\prime}$ with $d_{2}\left(E^{\prime} / K\right)=1$. Since $E(K)[2]=0$, it follows from (1) that either $\operatorname{rank}\left(E^{\prime}(K)\right)=1$ or $\operatorname{dim}_{\mathbf{F}_{2}} \amalg\left(E^{\prime} / K\right)[2]=1$. But Conjecture $\amalg_{2}(K)$ says that $\operatorname{dim}_{\mathbf{F}_{2}} \amalg\left(E^{\prime} / K\right)[2]$ is even, so $\operatorname{rank}\left(E^{\prime}(K)\right)=1$. By Lemma 5.5, all but finitely many of these twists have $E^{\prime}(K)_{\text {tors }}=0$, and this proves the corollary.

\section{Proof of Theorem 1.13 when $[L: K]=2$}

Proposition 6.1 Suppose $L / K$ is a quadratic extension. Then there is an elliptic curve $E / K$ such that $\operatorname{Gal}(K(E[2]) / K) \cong S_{3}$ and $d_{2}(E / K)+$ $d_{2}\left(E^{L} / K\right)$ is odd.

Proof We thank the referee for pointing out the following simple proof of this proposition.

Fix a prime $\mathfrak{p} \nmid 6$ that remains prime in $L / K$. Using Lemma 5.4, fix an elliptic curve $E$ over $K$ with $\operatorname{Gal}(K(E[2]) / K) \cong S_{3}$, with multiplicative reduction at $\mathfrak{p}$, and with $\operatorname{ord}_{\mathfrak{p}}\left(\Delta_{E}\right)=1$. Fix also a quadratic extension $M / K$ that is ramified at $\mathfrak{p}$, and split at all of the following places: all primes different from $\mathfrak{p}$ where $E$ has bad reduction, all primes above 2, all infinite places, and all places ramified in $L / K$.

Recall the local norm index $\delta_{v}(E, L / K)$ of Definition 2.6. By Kramer's congruence (Theorem 2.7) we have

$$
d_{2}(E / L)+d_{2}\left(E^{M} / L\right) \equiv \sum_{w} \delta_{w}(E, L M / L)(\bmod 2),
$$

summing over all places $w$ of $L$. We will show that the sum in (11) is odd. 
If $w$ divides $2 \infty$, or $w \neq \mathfrak{p}$ is a prime where $E$ has bad reduction, then $w$ splits in $L M / L$, so Lemma 2.10(i) shows that $\delta_{w}(E, L M / L)=0$. If $w$ is a prime where $E$ has good reduction and $w$ is unramified in $L M / L$, then $\delta_{w}(E, L M / L)=0$ by Lemma $2.10(\mathrm{v})$.

Suppose $w \nmid 2 \infty, E$ has good reduction at $w$, and $w$ ramifies in $L M / L$. Let $v$ denote the prime of $K$ below $w$. If $v$ splits in $L / K$ into two places $w, w^{\prime}$, then $\delta_{w}(E, L M / L)=\delta_{w^{\prime}}(E, L M / L)$ so the contribution $\delta_{w}(E, L M / L)+$ $\delta_{w^{\prime}}(E, L M / L)$ in (11) is even. If $v$ is inert in $L / K$, then either $E\left(K_{v}\right)[2]=0$, in which case $E\left(F_{w}\right)[2]=0$ as well, or $E\left(K_{v}\right)[2] \neq 0$, in which case $E\left(F_{w}\right)[2]=E[2]$. In either case Proposition 2.11 shows that $\delta(E, L M / L)=$ $\operatorname{dim}_{\mathbf{F}_{2}}\left(E\left(F_{w}\right)[2]\right)$ is even.

We conclude now from (11) that

$$
d_{2}(E / L)+d_{2}\left(E^{M} / L\right) \equiv \delta_{\mathfrak{p}}(E, L M / L)(\bmod 2) .
$$

Since $L_{\mathfrak{p}}$ is the unramified quadratic extension of $K_{\mathfrak{p}}, E$ has split multiplicative reduction over $L_{\mathfrak{p}}$. It follows from [11, Proposition 1] that $\delta_{\mathfrak{p}}(E, L M / L)=1$.

Therefore $d_{2}(E / L)+d_{2}\left(E^{M} / L\right)$ is odd. Replacing $E$ by $E^{M}$ if necessary, we may suppose that $d_{2}(E / L)$ is odd. Since $E(K)[2]=0$, we have $E(L)[2]=0$ as well, so $d_{2}(E / L) \equiv d_{2}(E / K)+d_{2}\left(E^{L} / K\right)(\bmod 2)$ by Lemma 2.5, and the proof is complete.

Theorem 6.2 Suppose $L / K$ is a quadratic extension of number fields. There is an elliptic curve $E$ over $K$ such that $d_{2}(E / K)=0$ and $d_{2}\left(E^{L} / K\right)=1$. In particular $\operatorname{rank}\left(E^{L}(K)\right)=\operatorname{rank}\left(E^{L}(L)\right)$, and if Conjecture $\amalg_{2}(K)$ holds then $\operatorname{rank}\left(E^{L}(K)\right)=\operatorname{rank}\left(E^{L}(L)\right)=1$.

Proof Fix an elliptic curve $A$ over $K$ satisfying the conclusion of Proposition 6.1: $\operatorname{Gal}(K(A[2]) / K) \cong S_{3}$ and $d_{2}(A / K), d_{2}\left(A^{L} / K\right)$ have opposite parity.

Now apply Proposition 5.1(ii) repeatedly (with $L^{\prime}=K$ ), twisting $A$ until we produce a twist $B$ with either $d_{2}(B / K)=0$ or $d_{2}\left(B^{L} / K\right)=0$. Switching $B$ and $B^{L}$ if necessary, we may suppose that $d_{2}(B / K)=0$.

Note that $d_{2}(B / K)$ and $d_{2}\left(B^{L} / K\right)$ still have opposite parity, so $d_{2}\left(B^{L} / K\right) \geq 1$. If $d_{2}\left(B^{L} / K\right)=1$ we stop. If $d_{2}\left(B^{L} / K\right)>1$ we apply Proposition 5.1(iii) and then Proposition 5.1(ii), to obtain at twist $C$ with $d_{2}(C / K)=0$ and $d_{2}\left(C^{L} / K\right)=d_{2}\left(B^{L} / K\right)-2$. Continuing in this way we eventually obtain a twist $E$ with $d_{2}(E / K)=0$ and $d_{2}\left(E^{L} / K\right)=1$.

We have $\operatorname{rank}(E(K))=0$, so

$$
\operatorname{rank}\left(E^{L}(L)\right)=\operatorname{rank}(E(K))+\operatorname{rank}\left(E^{L}(K)\right)=\operatorname{rank}\left(E^{L}(K)\right),
$$

and if Conjecture ${ }^{2} T_{2}(K)$ holds then $\operatorname{rank}\left(E^{L}(K)\right)=1$. 


\section{Two-descents over cyclic extensions of odd prime degree}

Fix for this section a number field $K$, and a cyclic extension $L / K$ of prime degree $p>2$. Let $G=\operatorname{Gal}(L / K)$. If $R$ is a commutative ring, let $R[G]^{0}$ denote the augmentation ideal in the group ring $R[G]$.

Since $|G|$ is odd, the group ring $\mathbf{F}_{2}[G]$ is an étale $\mathbf{F}_{2}$-algebra. Concretely, if we fix a generator of $G$ we have $G$-isomorphisms

$$
\mathbf{F}_{2}[G] \cong \mathbf{F}_{2}[X] /\left(X^{p}-1\right) \cong \mathbf{F}_{2} \oplus\left(\prod_{\pi} \mathbf{F}_{2}[X] / \pi(X)\right)
$$

where $\pi$ runs through the irreducible factors of $X^{p-1}+\cdots+1$ in $\mathbf{F}_{2}[X]$, and the chosen generator of $G$ acts on $\mathbf{F}_{2}[X]$ as multiplication by $X$. The submodule of $\mathbf{F}_{2}[G]$ corresponding to the summand $\mathbf{F}_{2}$ in (12) is $\mathbf{F}_{2}[G]^{G}$, and the submodule of $\mathbf{F}_{2}[G]$ corresponding to $\prod_{\pi} \mathbf{F}_{2}[X] / \pi(X)$ is the augmentation ideal $\mathbf{F}_{2}[G]^{0}$. Thus (12) corresponds to the decomposition (independent of choice of generator of $G$ )

$$
\mathbf{F}_{2}[G]=\mathbf{F}_{2}[G]^{G} \oplus \mathbf{F}_{2}[G]^{0}=\mathbf{F}_{2} \oplus\left(\bigoplus_{\mathbb{k} \in \Omega} \mathbb{k}\right)
$$

where $\Omega$ is the set of simple submodules of $\mathbf{F}_{2}[G]$ on which $G$ acts nontrivially.

If $B$ is an $\mathbf{F}_{2}[G]$-module, then $B \otimes \mathbf{F}_{2}[G] \mathbf{F}_{2}=B^{G}$, and we define

$$
B^{\text {new }}=B \otimes_{\mathbf{F}_{2}[G]} \mathbf{F}_{2}[G]^{0}=\bigoplus_{\mathbb{k} \in \Omega}\left(B \otimes_{\mathbf{F}_{2}[G]} \mathbb{k}\right) .
$$

This gives a canonical decomposition $B=B^{G} \oplus B^{\text {new }}$.

Suppose now that $E$ is an elliptic curve over $K$. The 2-Selmer group $\operatorname{Sel}_{2}(E / L)$ has a natural action of $\mathbf{F}_{2}[G]$. Since $|G|$ is odd, it is straightforward to check that $\operatorname{Sel}_{2}(E / L)^{G}=\operatorname{Sel}_{2}(E / K)$, so

$$
\operatorname{Sel}_{2}(E / L)=\operatorname{Sel}_{2}(E / K) \oplus \operatorname{Sel}_{2}(E / L)^{\text {new }} .
$$

For $\mathbb{k} \in \Omega$ we define a non-negative integer

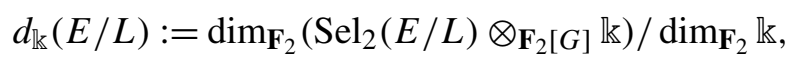

the multiplicity of $\mathbb{k}$ in the $\mathbf{F}_{2}[G]$-module $\operatorname{Sel}_{2}(E / L)$.

Remark 7.1 Our proof of Theorem 1.13 for $L / K$ goes as follows. We show that if $E$ satisfies the hypotheses of Theorem 1.6, then: 
(1) There is a twist $E^{\prime}$ of $E$ over $K$ such that $d_{\mathbb{k}}\left(E^{\prime} / L\right)=0$ for some $\mathbb{k}$ (see Proposition 7.4).

(2) For every $r \geq 0$, there is a twist $E^{\prime}$ of $E$ over $K$ such that $d_{2}\left(E^{\prime} / K\right)=r$ and $\operatorname{Sel}_{2}\left(E^{\prime} / L\right)^{\text {new }}=\operatorname{Sel}_{2}(E / L)^{\text {new }}$ (see Proposition 7.5). In other words, we can twist to get whatever size we want for the "old part" of Selmer, while keeping the "new part" of Selmer unchanged.

Replacing $E$ by a quadratic twist as necessary, by (1) we may assume $d_{\mathbb{k}}(E / L)=0$ for some $\mathbb{k}$. Then by (2) we may assume that both $d_{2}(E / K)=1$ and $d_{\mathbb{k}}(E / L)=0$. Since $d_{\mathbb{k}}(E / L)=0$ for some $\mathbb{k}$, we have $\operatorname{rank}(E(L))=$ $\operatorname{rank}(E(K))$ (see Lemma 7.2), and if Conjecture $\amalg_{2}(K)$ holds, then $\operatorname{rank}(E(K))=1$.

Lemma 7.2 Suppose $E$ is an elliptic curve over $K$. If $d_{\mathbb{k}}(E / L)=0$ for some $\mathbb{k} \in \Omega$, then $\operatorname{rank}(E(L))=\operatorname{rank}(E(K))$.

Proof Since $G$ is cyclic of prime order, it has only 2 irreducible rational representations, namely $\mathbf{Q}$ (the trivial representation) and the augmentation ideal $\mathbf{Q}[G]^{0}$. Therefore we have an isomorphism of $G$-modules

$$
E(L) \otimes \mathbf{Q} \cong \mathbf{Q}^{a} \times\left(\mathbf{Q}[G]^{0}\right)^{b}
$$

for some $a, b \geq 0$. Then $E(L)$ has a submodule isomorphic to $\left(\mathbf{Z}[G]^{0}\right)^{b}$, so $E(L) \otimes \mathbf{Z}_{2}$ has a direct summand isomorphic to $\left(\mathbf{Z}_{2}[G]^{0}\right)^{b}$, so $E(L) \otimes \mathbf{F}_{2}$ has a submodule isomorphic to $\left(\mathbf{F}_{2}[G]^{0}\right)^{b}$, which implies that $d_{\mathbb{k}}(E / L) \geq b$. Since $d_{\mathbb{k}}(E / L)=0$ we have $b=0$, and so $\operatorname{rank}(E(L))=\operatorname{rank}(E(K))=a$.

We will need the following $G$-equivariant version of Proposition 3.3.

Proposition 7.3 Suppose $F / K$ is a quadratic extension and the hypotheses of Proposition 3.3 are satisfied. Let $T$ be the set of primes of $K$ where $F / K$ is ramified, and let $T_{L}$ be the set of primes of $L$ above $T$.

(i) If the localization map

$$
\operatorname{loc}_{T_{L}}: \operatorname{Sel}_{2}(E / L)^{\text {new }} \rightarrow\left(\bigoplus_{\mathfrak{P} \in T_{L}} H_{\mathrm{f}}^{1}\left(L_{\mathfrak{P}}, E[2]\right)\right)^{\text {new }}
$$

is surjective, then there is an exact sequence

$$
\begin{aligned}
& 0 \longrightarrow \operatorname{Sel}_{2}\left(E^{F} / L\right)^{\text {new }} \longrightarrow \operatorname{Sel}_{2}(E / L)^{\text {new }} \\
& \stackrel{\operatorname{loc}_{T_{L}}}{\longrightarrow}\left(\bigoplus_{\mathfrak{P} \in T_{L}} H_{\mathrm{f}}^{1}\left(L_{\mathfrak{P}}, E[2]\right)\right)^{\text {new }} \longrightarrow 0 .
\end{aligned}
$$


(ii) Suppose that for every prime $\mathfrak{p} \in T, \mathfrak{p}$ is inert in $L / K$ and $E\left(K_{\mathfrak{p}}\right)[2] \neq 0$. Then $\operatorname{Sel}_{2}\left(E^{F} / L\right)^{\text {new }}=\operatorname{Sel}_{2}(E / L)^{\text {new }}$.

Proof The proof is identical to that of Proposition 3.3, using that the functor $B \mapsto B^{\text {new }}$ is exact on $\mathbf{F}_{2}[G]$-modules. As in the proof of Proposition 3.3, we have ( $G$-equivariant) exact sequences

$$
\begin{aligned}
& 0 \rightarrow \mathcal{S}_{T_{L}}^{\text {new }} \rightarrow \operatorname{Sel}_{2}(E / L)^{\text {new }} \stackrel{\operatorname{loc}_{T_{L}}}{\longrightarrow}\left(\bigoplus_{\mathfrak{P} \in T_{L}} H_{\mathrm{f}}^{1}\left(L_{\mathfrak{P}}, E[2]\right)\right)^{\text {new }} \\
& 0 \rightarrow \mathcal{S}_{T_{L}}^{\text {new }} \rightarrow \operatorname{Sel}_{2}\left(E^{F} / L\right)^{\text {new }} \rightarrow\left(\bigoplus_{\mathfrak{P} \in T_{L}} H_{\mathrm{f}}^{1}\left(L_{\mathfrak{P}}, E^{F}[2]\right)\right)^{\text {new }}
\end{aligned}
$$

either of which can be taken as the definition of $\mathcal{S}_{T_{L}}^{\text {new }}$. The proof of Proposition 3.3 showed that if $\operatorname{loc}_{T_{L}}$ is surjective, then the right-hand map of (14) is zero, and then (13) is the exact sequence of (i).

Suppose $\mathfrak{p} \in T$ is inert in $L / K$. Let $\operatorname{Frob}_{\mathfrak{p}} \in \operatorname{Gal}\left(K_{\mathfrak{p}}^{\text {ur }} / K_{\mathfrak{p}}\right)$ be the Frobenius of $\mathfrak{p}$, so Frob $b_{\mathfrak{P}}=\operatorname{Frob}_{\mathfrak{p}}^{p}$ is the Frobenius of the prime $\mathfrak{P}$ above $\mathfrak{p}$. Since $\mathfrak{p} \in T$, the hypotheses of Proposition 3.3 require that $E$ has good reduction at $\mathfrak{p}$, so by Lemma 2.2(ii) there is a commutative diagram with horizontal isomorphisms

$$
\begin{aligned}
H_{\mathrm{f}}^{1}\left(L_{\mathfrak{P}}, E[2]\right) & \sim \\
\operatorname{Res} \uparrow & \\
H_{\mathrm{f}}^{1}\left(K_{\mathfrak{p}}, E[2] /\left(\text { Frob }_{\mathfrak{P}}-1\right) E[2]\right. & \stackrel{\uparrow}{\longrightarrow} E[2] /\left(\text { Frob }_{\mathfrak{p}}-1\right) E[2] .
\end{aligned}
$$

If $E\left(K_{\mathfrak{p}}\right)[2] \neq 0$, then Frob $_{\mathfrak{p}}$ acts on $E[2]$ as an element of order 1 or 2, so Frob $\left._{\mathfrak{P}}\right|_{E[2]}=$ Frob $\left._{\mathfrak{p}}\right|_{E[2]}$ and the groups on the right have the same order. The left-hand vertical map is injective since $\left[L_{\mathfrak{P}}: K_{\mathfrak{p}}\right]$ is odd. Therefore the lefthand map is an isomorphism, so $G$ acts trivially on $H_{\mathrm{f}}^{1}\left(L_{\mathfrak{P}}, E[2]\right)$, and we have $H^{1}\left(L_{\mathfrak{P}}, E[2]\right)^{\text {new }}=0$.

If every $\mathfrak{p} \in T$ has these properties, then $\left(\bigoplus_{\mathfrak{P} \in T_{L}} H_{\mathfrak{f}}^{1}\left(L_{\mathfrak{P}}, E[2]\right)\right)^{\text {new }}=0$, so (ii) follows from (i).

Proposition 7.4 Suppose $E$ is an elliptic curve over $K$ satisfying the hypotheses of Theorem 1.6. If $d_{\mathbb{k}}(E / L)>0$ for every $\mathbb{k} \in \Omega$, then there is a quadratic twist $E^{\prime}$ of $E$ over $K$ such that

$$
d_{\mathbb{k}}\left(E^{\prime} / L\right)=d_{\mathbb{k}}(E / L)-1
$$

for every $\mathbb{k} \in \Omega$. 
Proof Let $\Delta$ be the discriminant of (some integral model of) $E$. Let $M:=$ $K(E[2])$, so $M$ is an $S_{3}$-extension of $K$ containing the quadratic extension $K(\sqrt{\Delta})$. Let $\Sigma$ be the set of all infinite places and all primes where $E$ has bad reduction.

Let $\mathfrak{d}$ be the (formal) product of all places in $\Sigma-\left\{v_{0}\right\}$, where $v_{0} \nmid 2$ is the distinguished place of Theorem 1.6, either real with $\Delta_{v_{0}}<0$, or of multiplicative reduction with ord $v_{0}(\Delta)$ odd. Let $K(8 \mathfrak{d})$ denote the ray class field of $K$ modulo 8d, and let $K[8 \mathfrak{d}]$ denote the maximal 2-power extension of $K$ in $K(8 \mathfrak{d})$. Note that $K(\sqrt{\Delta}) / K$ is ramified at $v_{0}$, but $L K[8 \mathfrak{d}] / K$ is unramified at $v_{0}$, so $M \cap L K[8 \mathfrak{d}]=K(\sqrt{\Delta}) \cap L K[8 \mathfrak{d}]=K$. Fix an element $\sigma \in G_{K}$, trivial on $L K$ [8d], whose projection to $\operatorname{Gal}(M L K[8 \mathfrak{d}] / L K[8 \mathfrak{d}]) \cong \mathrm{Gal}(M / K) \cong S_{3}$ has order 2. Since $\sigma$ has order 2 on $M$, we have $E[2] /(\sigma-1) E[2] \cong \mathbf{Z} / 2 \mathbf{Z}$.

Since $d_{\mathbb{k}}(E / L) \geq 1$ for every $\mathbb{k} \in \Omega$, it follows that $\operatorname{Sel}_{2}(E / L)^{\text {new }}$ has a submodule free of rank one over $\mathbf{F}_{2}[G]^{0}$. Let $C \subset \operatorname{Sel}_{2}(E / L)^{\text {new }}$ be such a submodule, fix an isomorphism $\eta: C \rightarrow \mathbf{F}_{2}[G]^{0}$, and define $\phi: C \rightarrow$ $E[2] /(\sigma-1) E[2]$ by $\phi(c)=f_{1}(\eta(c)) x$, where $f_{1}: \mathbf{F}_{2}[G] \rightarrow \mathbf{F}_{2}$ is projection onto the first coefficient, i.e., $f_{1}\left(\sum a_{g} g\right)=a_{1}$, and $x$ is the nonzero element of $E[2] /(\sigma-1) E[2]$.

By Lemma 3.5 we can find $\gamma \in G_{K}$ such that

- $\left.\gamma\right|_{L M K[80]}=\sigma$,

- $c(\gamma)=\phi(c)$ for all $c \in C$.

Let $N$ be a Galois extension of $K$ containing $M L K[8 \mathfrak{d}]$, large enough so that the restriction of $c$ to $N$ is zero. Let $\mathfrak{p}$ be a prime of $K$ where $E$ has good reduction, not dividing 2 , unramified in $L / K$, whose Frobenius in $\operatorname{Gal}(N / K)$ is in the conjugacy class of $\gamma$. Since $\left.\gamma\right|_{K[80]}=\left.\sigma\right|_{K[80]}=1$, and $[K(8 \mathfrak{d}): K[8 \mathfrak{d}]]$ is odd, there is an odd positive integer $h$ such that $\left.\gamma^{h}\right|_{K(8 \mathfrak{d})}=1$. Therefore $\mathfrak{p}^{h}$ is principal, with a generator $\pi \equiv 1(\bmod 8 \mathfrak{d})$, positive at all real embeddings different from $v_{0}$. Let $F=K(\sqrt{\pi})$. Then all places $v$ dividing 2 and all places in $\Sigma-\left\{v_{0}\right\}$ split in $F$, and $F / K$ is ramified only at $\mathfrak{p}$. Let $E^{\prime}$ be the quadratic twist of $E$ by $F$. We will show that $E^{\prime}$ has the desired properties.

We will apply Proposition 7.3. Let $T=\{\mathfrak{p}\}$, and $T_{L}$ the set of primes of $L$ above $\mathfrak{p}$. For every $\mathfrak{P} \in T_{L}$,

$$
\begin{aligned}
H_{\mathrm{f}}^{1}\left(L_{\mathfrak{P}}, E[2]\right) & =H^{1}\left(L_{\mathfrak{P}}^{\mathrm{ur}} / L_{\mathfrak{P}}, E[2]\right)=E[2] /\left(\text { Frob }_{\mathfrak{P}}-1\right) E[2] \\
& =E[2] /(\sigma-1) E[2]
\end{aligned}
$$

is a one-dimensional $\mathbf{F}_{2}$-vector space. Fix a prime of $N$ above $\mathfrak{p}$ whose Frobenius in $\operatorname{Gal}(N / K)$ is equal to $\gamma$, and let $\mathfrak{P}_{0}$ be the corresponding prime of $L$. Then $T_{L}=\left\{\mathfrak{P}_{0}^{\tau}: \tau \in G\right\}$, and Frob $_{\mathfrak{P}_{0}^{\tau} / \mathfrak{p}}(N / K)=\tau \gamma \tau^{-1}$. The localization 
map

$$
\operatorname{loc}_{T_{L}}: \operatorname{Sel}_{2}(E / L) \rightarrow \bigoplus_{\mathfrak{P} \in T_{L}} H_{\mathrm{f}}^{1}\left(L_{\mathfrak{P}}, E[2]\right) \cong \mathbf{F}_{2}[G] \otimes_{\mathbf{Z}}(E[2] /(\sigma-1) E[2])
$$

is given on $c \in C \subset \operatorname{Sel}_{2}(E / L)^{\text {new }}$ by

$$
\begin{aligned}
\operatorname{loc}_{T_{L}}(c) & =\sum_{\tau} \tau \otimes c\left(\tau \gamma \tau^{-1}\right)=\sum_{\tau} \tau \otimes c^{\tau^{-1}}(\gamma)=\sum_{\tau} \tau \otimes \phi\left(c^{\tau^{-1}}\right) \\
& =\sum_{\tau} \tau \otimes f_{1}\left(\tau^{-1} \eta(c)\right) x=\sum_{\tau} \tau \otimes f_{\tau}(\eta(c)) x=\eta(c) \otimes x
\end{aligned}
$$

where $f_{\tau}: \mathbf{F}_{2}[G] \rightarrow \mathbf{F}_{2}$ is the map $f_{\tau}\left(\sum a_{g} g\right)=a_{\tau}$. Since the image of $\eta$ is $\mathbf{F}_{2}[G]^{0}$, this shows that the localization map $C \rightarrow\left(\bigoplus_{\mathfrak{P} \in T_{L}} H_{\mathrm{f}}^{1}\left(L_{\mathfrak{P}}, E[2]\right)\right)^{\text {new }}$ is surjective. Now Proposition 7.3(i) shows that $\operatorname{Sel}_{2}\left(E^{F} / L\right)^{\text {new }}$ sits inside $\operatorname{Sel}_{2}(E / L)^{\text {new }}$ with cokernel containing a copy $\mathbf{F}_{2}[G]^{0}$, so $d_{\mathbb{k}}\left(E^{F} / L\right)<$ $d_{\mathbb{k}}(E / L)$ for every $\mathbb{k} \in \Omega$.

Proposition 7.5 Suppose $E$ is an elliptic curve over $K$ satisfying the hypotheses of Theorem 1.6. Then:

(i) There is a quadratic twist $E^{\prime}$ of $E / K$ such that $d_{2}\left(E^{\prime} / K\right)=d_{2}(E / K)+1$ and $\operatorname{Sel}_{2}\left(E^{\prime} / L\right)^{\text {new }}=\operatorname{Sel}_{2}(E / L)^{\text {new }}$.

(ii) If $\operatorname{Sel}_{2}(E / K) \neq 0$, then there is a quadratic twist $E^{\prime}$ of $E / K$ such that $d_{2}\left(E^{\prime} / K\right)=d_{2}(E / K)-1$ and $\operatorname{Sel}_{2}\left(E^{\prime} / L\right)^{\text {new }}=\operatorname{Sel}_{2}(E / L)^{\text {new }}$.

Proof Let $\Sigma$ be the set of all places $v \mid 2 \infty$ of $K$ and all $v$ of bad reduction, and let $v_{0}$ be the distinguished place of Theorem 1.6, either real with $\Delta_{v_{0}}<0$, or of multiplicative reduction with $\operatorname{ord}_{v_{0}}(\Delta)$ odd. By Proposition 5.1, for (i) or (ii) we can find a quadratic extension $F / K$ satisfying

- $d_{2}\left(E^{F} / K\right)=d_{2}(E / K)+1$ in (i), $d_{2}\left(E^{F} / K\right)=d_{2}(E / K)-1$ in (ii),

- all $v \in \Sigma-\left\{v_{0}\right\}$ split in $F / K$, and $v_{0}$ is unramified in $F / K$,

- $F / K$ is ramified at exactly one prime $\mathfrak{p}, \mathfrak{p} \nmid 2, \mathfrak{p}$ is inert in $L / K$, and $E\left(K_{\mathfrak{p}}\right)[2] \cong \mathbf{Z} / 2 \mathbf{Z}$.

By Proposition 7.3(ii) applied with $T=\{\mathfrak{p}\}, \operatorname{Sel}_{2}\left(E^{F} / L\right)^{\text {new }}=\operatorname{Sel}_{2}(E / L)^{\text {new }}$ in both cases.

Corollary 7.6 Suppose E/K satisfies the hypotheses of Theorem 1.6, and $r \geq 0$. Then there is a twist $E^{\prime}$ of $E$ such that

$$
d_{2}\left(E^{\prime} / K\right)=r, \quad \operatorname{rank}\left(E^{\prime}(L)\right)=\operatorname{rank}\left(E^{\prime}(K)\right) .
$$


Proof Using Proposition 7.4 repeatedly, we can find a twist $E^{\prime \prime}$ of $E$ such that $d_{\mathbb{k}}\left(E^{\prime \prime} / L\right)=0$ for at least one $\mathbb{k}$. Then applying Proposition 7.5 repeatedly, we can find another twist $E^{\prime}$ of $E$ such that $d_{\mathbb{k}}\left(E^{\prime} / L\right)=0$ and $d_{2}\left(E^{\prime} / K\right)=r$. Now the corollary follows from Lemma 7.2.

Proof of Theorem 1.13 Let $p=[L: K]$. If $p=2$, Theorem 1.13 is Theorem 6.2, so we may assume that $p$ is odd. By Lemma 5.4, we can find an elliptic curve $E$ over $K$ and a prime $\mathfrak{p} \nmid 2$ such that $E$ has multiplicative reduction at $\mathfrak{p}, \operatorname{ord}_{\mathfrak{p}}\left(\Delta_{E}\right)=1$, and $\operatorname{Gal}(K(E[2]) / K) \cong S_{3}$. Then $E$ satisfies the hypotheses of Theorem 1.6, so by Corollary 7.6, $E$ has a twist with the desired properties.

Remark 7.7 Assuming standard conjectures, there are noncyclic extensions $L / K$ for which the second part of Theorem 1.13 fails to hold. For example, suppose $F_{1}$ and $F_{2}$ are distinct quadratic extensions of $K$ such that every prime that ramifies in $F_{1} / K$ splits in $F_{2} / K$, and vice-versa. Let $L=F_{1} F_{2}$. It is not difficult to show that for every elliptic curve $E$ over $K$, the global root number of $E$ over $L$ is +1 . Thus (conjecturally) every elliptic curve $E$ over $K$ has even rank over $L$, so (conjecturally) there is no elliptic curve $E$ over $K$ with $\operatorname{rank}(E(L))=\operatorname{rank}(E(K))=1$.

\section{Proof of Theorem 1.2}

In this section we prove the following slightly stronger version of Theorem 1.2. The proof of Theorem 8.1 from Theorem 1.13 is due to Bjorn Poonen and Alexandra Shlapentokh. We thank them for allowing us to include their ideas here.

Theorem 8.1 Suppose $K$ is a number field and Conjecture $\mathrm{WT}_{2}(L)$ holds for all subfields $L$ of the Galois closure of $K / \mathbf{Q}$. Then Hilbert's Tenth Problem has a negative answer over the ring of integers of $K$.

Definition 8.2 Suppose that $R$ is a commutative ring with identity. Following [5,6], we say that a subset $D$ of $R$ is diophantine over $R$ if there is a finite set of polynomials $f_{1}, \ldots, f_{k} \in R\left[X, Y_{1}, \ldots, Y_{m}\right]$ for some $m$ such that for every $x \in R$,

$$
\begin{aligned}
x \in D \Longleftrightarrow \quad & \text { for } 1 \leq i \leq k \text { there are } y_{1, i}, \ldots, y_{m, i} \in R \\
& \text { such that } f_{i}\left(x, y_{1, i}, \ldots, y_{m, i}\right)=0 \text { for } 1 \leq i \leq k .
\end{aligned}
$$

Lemma 8.3 [6] Suppose $K \subset L$ are number fields. Then:

(i) If $D_{1}, D_{2} \subset \mathcal{O}_{L}$ are diophantine over $\mathcal{O}_{L}$, then so is $D_{1} \cap D_{2}$. 
(ii) If $D \subset \mathcal{O}_{K}$ is diophantine over $\mathcal{O}_{K}$, and $\mathcal{O}_{K}$ is diophantine over $\mathcal{O}_{L}$, then $D$ is diophantine over $\mathcal{O}_{L}$.

(iii) If $\mathbf{Z}$ is diophantine over $\mathcal{O}_{L}$, then $\mathbf{Z}$ is diophantine over $\mathcal{O}_{K}$.

Proof This is Proposition 1(a), (c), and (d) of [6].

Corollary 8.4 Suppose $L / K$ is a cyclic extension of number fields. If Conjecture $\amalg_{2}(F)$ holds for all subfields $F \subset L$, then $\mathcal{O}_{K}$ is diophantine over $\mathcal{O}_{L}$.

Proof We have $K=K_{0} \subset K_{1} \subset \cdots \subset K_{n}=L$, where each $K_{i+1} / K_{i}$ is cyclic of prime degree. If Conjecture $\mathrm{UT}_{2}\left(K_{i}\right)$ holds for every $i$, then by Theorem 1.13 for every $i$ there is an elliptic curve $E / K_{i}$ such that $\operatorname{rank}\left(E\left(K_{i}\right)\right)=$ $\operatorname{rank}\left(E\left(K_{i+1}\right)\right)=1$. By Theorem 1 of [20], it follows that $\mathcal{O}_{K_{i}}$ is diophantine over $\mathcal{O}_{K_{i+1}}$. Now the corollary follows from Lemma 8.3(ii) by induction.

Proof of Theorem 8.1 Fix a number field $K$, and let $L$ be the Galois closure of $K / \mathbf{Q}$. For every $g \in \operatorname{Gal}(L / \mathbf{Q})$, let $L^{\langle g\rangle}$ denote the fixed field of $g$ in $L$. Then $L / L^{\langle g\rangle}$ is cyclic, so $\mathcal{O}_{L}\langle g\rangle$ is diophantine over $\mathcal{O}_{L}$ by Corollary 8.4. But then by Lemma 8.3(i), $\bigcap_{g} \mathcal{O}_{L}\langle g\rangle=\mathcal{O}_{L}^{\mathrm{Gal}(L / \mathbf{Q})}=\mathbf{Z}$ is diophantine over $\mathcal{O}_{L}$, so by Lemma 8.3(iii), $\mathbf{Z}$ is diophantine over $\mathcal{O}_{K}$. Now the theorem follows from Matiyasevich's Theorem [12].

\section{Elliptic curves with constant parity}

In this section we discuss briefly the phenomenon of "constant parity".

Definition 9.1 Suppose $E$ is an elliptic curve defined over a number field $K$. We will say that $E / K$ has constant 2-Selmer parity if the parity of $d_{2}\left(E^{F} / K\right)$ is constant as $F$ ranges over all quadratic extensions of $K$, i.e., if $d_{2}\left(E^{F} / K\right) \equiv d_{2}(E / K)(\bmod 2)$ for all quadratic extensions $F / K$.

Similarly, we can say that $E$ has constant Mordell-Weil parity if the parity of $\operatorname{rank}\left(E^{F}(K)\right)$ is independent of the quadratic extension $F / K$, and $E$ has constant analytic parity if the global root number of $E^{F} / K$ is independent of $F$. Standard conjectures imply that all three notions of constant parity are the same.

Example 9.2 Suppose $E$ has complex multiplication by an imaginary quadratic field $k \subset K$. Then $E$ has constant (even) 2-Selmer parity, constant (even) Mordell-Weil parity, and constant (even) analytic parity.

The question of constant analytic parity was studied by Dokchitser and Dokchitser in [7]. They proved the following. 
Theorem 9.3 (Theorem 1 of [7]) An elliptic curve E over a number field $K$ has constant analytic parity if and only if $K$ is totally imaginary and $E$ acquires good reduction over an abelian extension of $K$.

The following example from [7] shows that constant parity can be odd.

Example 9.4 Suppose $K$ is totally imaginary, $E / K$ has good reduction everywhere, and $[K: \mathbf{Q}] / 2$ is odd. Then $E / K$ has constant odd analytic parity (see [21, Theorem 2(i) and Proposition 8(i)]).

This applies in particular to the elliptic curve

$$
E: y^{2}+x y=x^{3}+x^{2}-2 x-7
$$

(labeled 121C1 in Cremona's tables) and $K$ the splitting field of $x^{3}-11$.

From now on we will only consider constant 2-Selmer parity. The following theorem will be proved at the end of this section.

Theorem 9.5 If $E / K$ has constant 2-Selmer parity, then $K$ is totally imaginary and $E$ has no primes of multiplicative reduction.

Definition 9.6 Suppose $E$ is an elliptic curve defined over a local field $K$. If $F$ is a quadratic extension of $K$ (or $F=K$ ), define

$$
\delta(E, F / K)=\operatorname{dim}_{\mathbf{F}_{2}} E(K) / \mathbf{N}_{F / K} E(F) .
$$

We will say that $E / K$ has constant local parity if $\delta(E, F / K)$ is even for every quadratic extension $F / K$.

If $D \in K^{\times} /\left(K^{\times}\right)^{2}$, we will say that $E / K$ has $D$-parity if

$$
\delta(E, F / K) \text { is even } \Longleftrightarrow D \in \mathbf{N}_{F / K} F^{\times} .
$$

Note that if $D$ is a square in $K^{\times}$, then $E / K$ has $D$-parity if and only if it has constant local parity.

Lemma 9.7 Suppose $E$ is an elliptic curve defined over a local field $K$, and $\Delta_{E} \in K^{\times} /\left(K^{\times}\right)^{2}$ is its discriminant.

(i) If $v$ is nonarchimedean with residue characteristic different from 2, and $E$ has good reduction, then $E$ has $\Delta_{E}$-parity.

(ii) If $K$ is nonarchimedean and $E$ has multiplicative reduction, then $E$ does not have $\Delta_{E}$-parity.

(iii) If $K=\mathbf{R}$, then $E$ does not have $\Delta_{E}$-parity. 
Proof Assertions (i), (ii), and (iii) are [13, Corollary 4.4] and [11, Proposition 3], [11, Propositions 1 and 2], and [11, Proposition 6], respectively.

For the rest of this section, fix an elliptic curve $E$ defined over a number field $K$, and let $\Delta_{E}$ be the discriminant of some model of $E$.

\section{Theorem 9.8}

(i) If $E / K_{v}$ has constant local parity for every place $v$ of $K$, then $E / K$ has constant 2-Selmer parity.

(ii) $E / K$ has constant 2-Selmer parity if and only if $E / K_{v}$ has $\Delta_{E}$-parity for every $v$.

Proof Suppose $F$ is a quadratic extension of $K$. Kramer's congruence (Theorem 2.7) says

$$
d_{2}\left(E^{F} / K\right) \equiv d_{2}(E / K)+\sum_{v} \delta\left(E, F_{v} / K_{v}\right)(\bmod 2)
$$

where $F_{v}$ is the completion of $F$ at some place above $v$. Assertion (i) follows directly from this.

Now suppose $E / K_{v}$ has $\Delta_{E}$-parity for every $v$. Then, if $\tau$ is the nontrivial automorphism of $\operatorname{Gal}(F / K)$,

$$
\tau^{\delta\left(E, F_{v} / K_{v}\right)}=\left[\Delta_{E}, F_{v} / K_{v}\right]
$$

where $\left[\cdot, F_{v} / K_{v}\right]$ is the local Artin symbol. The global reciprocity law shows that $\prod_{v}\left[\Delta_{E}, F_{v} / K_{v}\right]=1$, so $\sum_{v} \delta\left(E, F_{v} / K_{v}\right)$ is even and it follows from (16) that $E / K$ has constant 2-Selmer parity.

Finally, suppose that for some $v_{0}, E / K_{v_{0}}$ does not have $\Delta_{E}$-parity. By Lemma 9.7(i), $E / K_{v}$ has $\Delta_{E}$-parity for almost all $v$. Fix a quadratic extension $F / K$ such that

- $\tau^{\delta\left(E, F_{v_{0}} / K_{v_{0}}\right)}=\tau \cdot\left[\Delta_{E}, F_{v_{0}} / K_{v_{0}}\right]$,

- every $v \neq v_{0}$ where $E / K_{v}$ does not have $\Delta_{E}$-parity splits in $F / K$.

Then $\tau^{\delta\left(E, F_{v} / K_{v}\right)}=\left[\Delta_{E}, F_{v} / K_{v}\right]$ for every $v \neq v_{0}$, so

$$
\tau^{\sum_{v} \delta\left(E, F_{v} / K_{v}\right)}=\tau \cdot \prod_{v}\left[\Delta_{E}, F_{v} / K_{v}\right]=\tau,
$$

so by (16), $d_{2}(E / K)$ and $d_{2}\left(E^{F} / K\right)$ have opposite parity.

Proof of Theorem 9.5 Theorem 9.5 follows directly from Theorem 9.8(ii) and Lemma 9.7(ii, iii). 
Corollary 9.9 If $\Delta_{E}$ is a square, then $E / K$ has constant 2-Selmer parity if and only if $E / K_{v}$ has constant local parity for every $v$.

\section{Proof This is immediate from Theorem 9.8(ii).}

Acknowledgements The authors would like to thank Bjorn Poonen for asking the questions that led to this work. They also thank Poonen and Alexandra Shlapentokh for explaining how Theorem 1.13 implies Theorem 1.2, and for allowing us to describe their proof in Sect. 8.

Open Access This article is distributed under the terms of the Creative Commons Attribution Noncommercial License which permits any noncommercial use, distribution, and reproduction in any medium, provided the original author(s) and source are credited.

\section{References}

1. Cassels, J.W.S.: Arithmetic on an elliptic curve. In: Proc. Internat. Congr. Mathematicians, Stockholm, 1962, pp. 234-246. Inst. Mittag-Leffler, Djursholm (1963)

2. Cassels, J.W.S.: Arithmetic on curves of genus 1. VIII. On conjectures of Birch and Swinnerton-Dyer. J. Reine Angew. Math. 217, 180-199 (1965)

3. Chang, S.: On the arithmetic of twists of superelliptic curves. Acta Arith. 124, 371-389 (2006)

4. Chang, S.: Quadratic twists of elliptic curves with small Selmer rank. Preprint available at http://arxiv.org/abs/0809.5019

5. Denef, J.: Diophantine sets over algebraic integer rings. II. Trans. Am. Math. Soc. 257, 227-236 (1980)

6. Denef, J., Lipshitz, L.: Diophantine sets over some rings of algebraic integers. J. Lond. Math. Soc. 18, 385-391 (1978)

7. Dokchitser, T., Dokchitser, V.: Elliptic curves with all quadratic twists of positive rank. Acta Arith. 137, 193-197 (2009)

8. Eisenträger, K.: Hilbert's tenth problem and arithmetic geometry. Ph.D. thesis, UC Berkeley (2003)

9. Gouvêa, F., Mazur, B.: The square-free sieve and the rank of elliptic curves. J. Am. Math. Soc. 4, 1-23 (1991)

10. Heath-Brown, D.R.: The size of Selmer groups for the congruent number problem II. Invent. Math. 118, 331-370 (1994)

11. Kramer, K.: Arithmetic of elliptic curves upon quadratic extension. Trans. Am. Math. Soc. 264, 121-135 (1981)

12. Matiyasevich, Y.V.: The Diophantineness of enumerable sets. Dokl. Akad. Nauk SSSR 191, 279-282 (1970)

13. Mazur, B.: Rational points of abelian varieties with values in towers of number fields. Invent. Math. 18, 183-266 (1972)

14. Mazur, B., Rubin, K.: Kolyvagin systems, Mem. Am. Math. Soc., vol. 799 (2004)

15. Mazur, B., Rubin, K.: Finding large Selmer rank via an arithmetic theory of local constants. Ann. Math. 166, 581-614 (2007)

16. Merel, L.: Bornes pour la torsion des courbes elliptiques sur les corps de nombres. Invent. Math. 124, 437-449 (1996)

17. Milne, J.S.: Arithmetic Duality Theorems. Perspectives in Math., vol. 1. Academic Press, San Diego (1986)

18. Ono, K.: Nonvanishing of quadratic twists of modular $L$-functions and applications to elliptic curves. J. Reine Angew. Math. 533, 81-97 (2001) 
19. Ono, K., Skinner, C.: Non-vanishing of quadratic twists of modular $L$-functions. Invent. Math. 134, 651-660 (1998)

20. Poonen, B.: Using elliptic curves of rank one towards the undecidability of Hilbert's tenth problem over rings of algebraic integers. In: Algorithmic Number Theory, Sydney, 2002. Lecture Notes in Comput. Sci., vol. 2369, pp. 33-42. Springer, Berlin (2002)

21. Rohrlich, D.: Galois theory, elliptic curves, and root numbers. Compos. Math. 100, 311349 (1996)

22. Rubin, K.: Euler Systems. Annals of Math. Studies, vol. 147. Princeton University Press, Princeton (2000)

23. Serre, J.-P.: Divisibilité de certaines fonctions arithmétiques. Enseign. Math. 22, 227-260 (1976)

24. Shlapentokh, A.: Hilbert's tenth problem over number fields, a survey. In: Hilbert's Tenth Problem: Relations with Arithmetic and Algebraic Geometry, Ghent, 1999. Contemp. Math., vol. 270, pp. 107-137. Am. Math. Soc., Providence (2000)

25. Silverman, J.H.: The Arithmetic of Elliptic Curves. Graduate Texts in Mathematics, vol. 106. Springer, New York (1986)

26. Skorobogatov, A., Swinnerton-Dyer, P.: 2-descent on elliptic curves and rational points on certain Kummer surfaces. Adv. Math. 198, 448-483 (2005)

27. Swinnerton-Dyer, H.P.F.: The effect of twisting on the 2-Selmer group. Math. Proc. Cambridge Philos. Soc. 145, 513-526 (2008)

28. Tate, J.: Duality theorems in Galois cohomology over number fields. In: Proc. Intern. Congr. Math., Stockholm, pp. 234-241 (1962)

29. Wintner, A.: On the prime number theorem. Am. J. Math. 64, 320-326 (1942) 